\title{
Prestellar and protostellar cores in Orion B9 ${ }^{\star}$
}

\author{
O. Miettinen ${ }^{1}$, J. Harju ${ }^{1}$, L. K. Haikala ${ }^{1}$, J. Kainulainen ${ }^{1,2}$, and L. E. B. Johansson ${ }^{3}$ \\ 1 Observatory, PO Box 14, 00014 University of Helsinki, Finland \\ e-mail: oskari.miettinen@helsinki.fi \\ 2 TKK/Metsähovi Radio Observatory, Metsähovintie 114, 02540 Kylmälä, Finland \\ 3 Onsala Space Observatory, 43992 Onsala, Sweden
}

Received 17 December 2008 / Accepted 1 April 2009

ABSTRACT

\begin{abstract}
Context. Dense molecular cores are studied to gain insight into the processes causing clouds to fragment and form stars. In this study, we concentrate on a region that is assumed to represent an early stage of clustered star-formation in a giant molecular cloud. Aims. We aim to determine the properties and spatial distribution of dense cores in the relatively quiescent Ori B9 cloud, and to estimate their ages and dynamical timescales.

Methods. The cloud was mapped in the $870 \mu \mathrm{m}$ continuum with APEX/LABOCA, and selected positions were observed in the lines of $\mathrm{N}_{2} \mathrm{H}^{+}$and $\mathrm{N}_{2} \mathrm{D}^{+}$using IRAM-30 m. These were used together with our previous $\mathrm{H}_{2} \mathrm{D}^{+}$observations to derive the degree of deuteration and some other chemical characteristics. Archival far-infrared Spitzer/MIPS maps were combined with the LABOCA map to distinguish between prestellar and protostellar cores, and to estimate the evolutionary stages of protostars.

Results. Twelve dense cores were detected at $870 \mu \mathrm{m}$ continuum in the Ori B9 cloud. The submm cores constitute $\sim 4 \%$ of the total mass of the Ori B9 region. There is an equal number of prestellar and protostellar cores. Two of the submm sources, which we call SMM 3 and SMM 4, are previously unknown Class 0 candidates. There is a high likelihood of the core masses and mutual separations representing the same distributions as observed in other parts of Orion. We found a moderate degree of deuteration in $\mathrm{N}_{2} \mathrm{H}^{+}(0.03-0.04)$. There is, furthermore, evidence of $\mathrm{N}_{2} \mathrm{H}^{+}$depletion in the core SMM 4. These features suggest that the cores have reached chemical maturity. We derive a relatively high degree of ionization $\left(\sim 10^{-7}\right)$ in the clump associated with IRAS 05405-0117. The ambipolar diffusion timescales for two of the cores are $~ 70-100$ times longer than the free-fall time.

Conclusions. The distribution and masses of dense cores in Ori B9 are similar to those observed in more active regions of Orion, where the statistical core properties have been explained by turbulent fragmentation. The 50/50 proportions of prestellar and protostellar cores imply that the duration of the prestellar phase is comparable to the free-fall time. However, on the basis of chemical data of the IRAS 05405-0117 region, this timescale could be questioned. A possible explanation is that this survey samples only the densest, i.e., dynamically most advanced cores.
\end{abstract}

Key words. stars: formation - ISM: clouds - ISM: molecules - radio continuum: ISM - radio lines: ISM - submillimeter

\section{Introduction}

Most stars form in clusters and smaller groups in the densest parts of giant molecular clouds (GMCs). The fragmentation of molecular clouds results in dense filaments that contain still denser cores. These cold star-forming cores are detected most effectively using far-infrared (FIR) and submillimetre (submm) dust continuum. By studying their physical and chemical characteristics, one hopes to understand the conditions leading to protostellar collapse and the timescale related to this process. Furthermore, the distribution and spacing of dense cores place constraints on the fragmentation mechanisms (e.g., turbulent fragmentation and ambipolar diffusion) and the possible interaction between newly born stars and their surroundings (e.g., Megeath et al. 2008). The estimates of the core masses derived from dust continuum data can also be used to examine the possible connection between the mass distribution of dense cores and the stellar initial mass function (IMF), a question of consider-

* This publication is based on data acquired with the IRAM $30 \mathrm{~m}$ telescope and the Atacama Pathfinder Experiment (APEX). IRAM is supported by INSU/CNRS (France), MPG (Germany), and IGN (Spain). APEX is a collaboration between the Max-Planck-Institut für Radioastronomie, the European Southern Observatory, and the Onsala Space Observatory. able interest (e.g., Simpson et al. 2008; Swift \& Williams 2008; Goodwin et al. 2008; Rathborne et al. 2009).

Parameters affecting the cloud dynamics, such as the degree of ionization and the abundances of various positive ions are chemically related to deuterium fractionation and depletion of heavy species (e.g., CO). Besides being important to the core dynamics by means of magnetic support and molecular-line cooling, these parameters depend on the core history and characterise its present evolutionary stage. For example, substantial CO depletion and deuterium enrichment are supposed to be a characteristic of prestellar cores in the pivotal stage before collapse (Caselli et al. 1999; Bacmann et al. 2002; Lee et al. 2003). Observations (Tafalla et al. 2002, 2004, 2006) and some theoretical models (Bergin \& Langer 1997; Aikawa et al. 2005) however, suggest that $\mathrm{N}$-containing species such as the chemically closely-related nitrogen species $\mathrm{N}_{2} \mathrm{H}^{+}$and $\mathrm{NH}_{3}$ (and their deuterated isotopologues), remain in the gas phase at densities for which $\mathrm{CO}$ and other $\mathrm{C}$-containing molecules are already depleted (e.g., Flower et al. 2005, 2006b, and references therein). They are therefore considered useful spectroscopic tracers of prestellar cores and the envelopes of protostellar cores. However, there is some evidence that $\mathrm{N}_{2} \mathrm{H}^{+}$finally freezes out at densities $n\left(\mathrm{H}_{2}\right)=$ several $\times 10^{5}$ to $\gtrsim 10^{6} \mathrm{~cm}^{-3}$ (e.g., Bergin et al. 2002; Pagani et al. 2005, 2007). In contrast to this, $\mathrm{NH}_{3}$ abundance 
Table 1. Observational parameters.

\begin{tabular}{|c|c|c|c|c|c|c|c|c|c|c|}
\hline Molecule & Transition & $\begin{array}{c}\text { Frequency } \\
{[\mathrm{GHz}]}\end{array}$ & Instrument & $\overline{F_{\text {eff }}}$ & $\eta_{\mathrm{MB}}{ }^{a}$ & $\begin{array}{c}\mathrm{Re} \\
{\left[{ }^{\prime \prime}\right]}\end{array}$ & $\begin{array}{l}\text { olution } \\
{\left[\mathrm{km} \mathrm{s}^{-1}\right]}\end{array}$ & $\begin{array}{c}F_{\text {throw }} \\
{[\mathrm{MHz}]}\end{array}$ & $\begin{array}{l}T_{\text {sys }} \\
{[\mathrm{K}]}\end{array}$ & Obs. date \\
\hline $\mathrm{N}_{2} \mathrm{H}^{+}$ & $J=1-0$ & $93.173777^{b}$ & $30 \mathrm{~m} / \mathrm{AB} 100$ & 0.95 & 0.80 & 26.4 & 0.064 & 7.9 & $150-190$ & 18-20 May 2007 \\
\hline $\mathrm{N}_{2} \mathrm{D}^{+}$ & $J=2-1$ & $154.217154^{c}$ & $30 \mathrm{~m} / \mathrm{CD} 150$ & 0.93 & 0.73 & 16 & 0.038 & 15.8 & $290-340$ & 18-20 May 2007 \\
\hline \multicolumn{3}{|c|}{ Continuum at $870 \mu \mathrm{m}$} & APEX/LABOCA & 0.97 & 0.73 & 18.6 & - & - & - & 4 Aug. 2007 \\
\hline
\end{tabular}

${ }^{a} \eta_{\mathrm{MB}}=B_{\text {eff }} / F_{\text {eff }}$, where $B_{\text {eff }}$ and $F_{\text {eff }}$ are the beam and forward efficiencies, respectively. ${ }^{b}$ Frequency taken from Caselli et al. (1995). ${ }^{c}$ Frequency taken from Gerin et al. (2001).

appears to increase toward the centres of e.g., L1498 and L1517B (Tafalla et al. 2002, 2004). Similar results were found by Crapsi et al. (2007) using interferometric observations toward L1544.

\subsection{Ori B9}

Most molecular material in the Orion complex is concentrated in the Orion A and B clouds. Star formation in Orion B (L1630) takes place mainly in four clusters, NGC 2023, NGC 2024, NGC 2068, and NGC 2071 (e.g., Lada 1992; Launhardt et al. 1996). The Orion B South cloud, which encompasses the star-forming regions NGC 2023/2024, is the only site of $\mathrm{O}$ and $\mathrm{B}$ star formation in Orion B (see, e.g., Nutter \& Ward-Thompson 2007, hereafter NW07). Apart from the above-mentioned four regions, only single stars or small groups of low- to intermediate-mass stars are currently forming in Orion B (Launhardt et al. 1996).

Ori B9 is located in the central part of Orion B and is a relatively isolated cloud at a projected distance of $\sim 40^{\prime}(5.2 \mathrm{pc}$ at $450 \mathrm{pc}^{1}$ ) northeast of its closest star cluster NGC 2024 (Caselli \& Myers 1995), which is the most prominent region of current star formation in Orion B. Ori B9 has avoided previous (sub)mm mappings, which have concentrated on the well-known active regions in the northern and southern part of the GMC (NW07 and references therein).

In this paper, we present submm continuum mapping of the Ori B9 cloud with LABOCA on APEX, and spectral line observations towards three $\mathrm{N}_{2} \mathrm{H}^{+}$peaks found by Caselli \& Myers (1994) in the clump associated with the low-luminosity FIR source IRAS 05405-0117 (see Fig. 2 in Caselli \& Myers 1994) ${ }^{2}$. This source has the narrowest CS linewidth $\left(0.48 \mathrm{~km} \mathrm{~s}^{-1}\right)$ in the Lada et al. (1991) survey, and the narrowest $\mathrm{NH}_{3}$ linewidth (average linewidth is $0.29 \mathrm{~km} \mathrm{~s}^{-1}$ ) in the survey by Harju et al. (1993). A kinetic temperature of $10 \mathrm{~K}$ was derived from ammonia in this region.

We previously detected the $\mathrm{H}_{2} \mathrm{D}^{+}$ion towards two of the $\mathrm{N}_{2} \mathrm{H}^{+}$peaks (Harju et al. 2006). These detections suggest that 1) the degree of molecular depletion is high and 2) the ortho:para ratio of $\mathrm{H}_{2}$ is low, and thus the cores should have reached an evolved chemical stage. The high density and low temperature may have caused CO depletion. This possibility is supported by the clump associated with IRAS $05405-0117$ not being extremely conspicuous in the CO map of Caselli \& Myers (1995).

In the present study, we determine the properties and spatial distribution of dense cores in the Ori B9 cloud. We also derive the degree of deuteration and ionization degree within the clump associated with IRAS 05405-0117.

\footnotetext{
1 We assume a distance to the Orion star-forming regions of $450 \mathrm{pc}$.

2 The $\mathrm{N}_{2} \mathrm{H}^{+}(1-0)$ map of Caselli \& Myers (1994) shows two separated gas condensations of $\sim 0.1 \mathrm{pc}$ in size. The southern condensation has a weak subcomponent. The positions of our molecular-line observations are given in Table 1 of Harju et al. (2006).
}

The observations and data-reduction procedures are described in Sect. 2. The observational results are presented in Sect. 3. In Sect. 4, we describe the methods used to derive the physical and chemical properties of the observed sources. In Sect. 5, we discuss the results of our study, and in Sect. 6, we summarise our main conclusions.

\section{Observations and data reduction}

\subsection{Molecular lines: $\mathrm{N}_{2} \mathrm{H}^{+}$and $\mathrm{N}_{2} \mathrm{D}^{+}$}

The spectral-line observations towards the three abovementioned $\mathrm{N}_{2} \mathrm{H}^{+}$peaks were performed with the IRAM $30 \mathrm{~m}$ telescope on Pico Veleta, Spain, on May 18-20, 2007. The spectra were centred on the frequencies of the strongest $\mathrm{N}_{2} \mathrm{H}^{+}(1-0)$ and $\mathrm{N}_{2} \mathrm{D}^{+}(2-1)$ hyperfine components. We used the following rest-frame frequencies: $93173.777 \mathrm{MHz}\left(\mathrm{N}_{2} \mathrm{H}^{+}\left(J F_{1} F=123 \rightarrow\right.\right.$ 012), Caselli et al. 1995) and $154217.154 \mathrm{MHz}\left(\mathrm{N}_{2} \mathrm{D}^{+}(234 \rightarrow\right.$ 123), Gerin et al. 2001). Both Dore et al. (2004) and Pagani et al. (2009b) refined the $\mathrm{N}_{2} \mathrm{H}^{+}$and $\mathrm{N}_{2} \mathrm{D}^{+}$line frequencies. The slight differences between the "new" and "old" frequencies have, however, no practical effect on the radial velocities or other parameters derived here. The observations were performed in frequency-switching mode with the frequency throw set to 7.9 $\mathrm{MHz}$ for the $3 \mathrm{~mm}$ lines and $15.8 \mathrm{MHz}$ for the $2 \mathrm{~mm}$ lines.

As the spectral backend, we used the VESPA (Versatile Spectrometer Assembly) facility autocorrelator, which has a bandwidth of $20 \mathrm{MHz}$ and a channel width of $10 \mathrm{kHz}$. The lines were observed in two polarisations using the (AB) $100 \mathrm{GHz}$ and (CD) $150 \mathrm{GHz}$ receivers. The horizontal polarisation at higher frequency (D150) turned out to be very noisy and was thus excluded from the reduction. The channel width used corresponds to $0.032 \mathrm{~km} \mathrm{~s}^{-1}$ and $0.019 \mathrm{~km} \mathrm{~s}^{-1}$ at the observed frequencies of $\mathrm{N}_{2} \mathrm{H}^{+}(1-0)$ and $\mathrm{N}_{2} \mathrm{D}^{+}(2-1)$, respectively. The halfpower beamwidth (HPBW) and the main-beam efficiency, $\eta_{\mathrm{MB}}$, are $26 . ' 4$ and 0.80 , respectively, at $93 \mathrm{GHz}$, and $16^{\prime \prime}$ and 0.73 , respectively, at $154 \mathrm{GHz}$.

Calibration was achieved by the chopper-wheel method. The pointing and focus were checked regularly towards Venus and several quasars. Pointing accuracy was estimated to be superior to 4-6". The single-sideband (SSB) system temperatures were $\sim 150-190 \mathrm{~K}$ at $93 \mathrm{GHz}$ and $\sim 290-340 \mathrm{~K}$ at $154 \mathrm{GHz}$. We reached an rms sensitivity in antenna temperature units of about $0.03 \mathrm{~K}$ in $\mathrm{N}_{2} \mathrm{H}^{+}(1-0)$ and about $0.05-0.07 \mathrm{~K}$ in $\mathrm{N}_{2} \mathrm{D}^{+}(2-1)$. The observational parameters are listed in Table 1.

The CLASS programme, which is part of the GAG software developed at the IRAM and the Observatoire de Grenoble ${ }^{3}$, was used to complete the reduction. Third-order polynomial baselines were subtracted from the individual $\mathrm{N}_{2} \mathrm{H}^{+}(1-0)$ spectra before and after folding them. From each individual $\mathrm{N}_{2} \mathrm{D}^{+}(2-1)$ spectra, the fourth-order polynomial baselines were subtracted

\footnotetext{
${ }^{3}$ http://www.iram.fr/IRAMFR/GILDAS
} 
before folding. Finally, the summed spectra were Hanningsmoothed yielding the velocity resolutions of $0.064 \mathrm{~km} \mathrm{~s}^{-1}$ for $\mathrm{N}_{2} \mathrm{H}^{+}(1-0)$ and $0.038 \mathrm{~km} \mathrm{~s}^{-1}$ for $\mathrm{N}_{2} \mathrm{D}^{+}(2-1)$. We fitted the lines using the hyperfine structure fitting method of the CLASS programme. This method assumes that all the hyperfine components have the same excitation temperature and width $\left(T_{\text {ex }}\right.$ and $\Delta v$, respectively), and that their separations and relative line strengths are fixed to the values given in Caselli et al. (1995), Gerin et al. (2001), and Daniel et al. (2006). Besides $T_{\text {ex }}$ and $\Delta v$, this method provides an estimate of the total optical depth, $\tau_{\text {tot }}$, i.e., the sum of the peak optical depths of the hyperfine components. These parameters can be used to estimate the column density of the molecule.

\subsection{Submillimetre continuum}

The $870 \mu \mathrm{m}$ continuum observations toward the Ori B9 cloud were carried out on 4 August 2007 with the 295 channel bolometer array LABOCA (Large APEX Bolometer Camera) on APEX. The LABOCA central frequency was about $345 \mathrm{GHz}$ and the bandwidth was about $60 \mathrm{GHz}$. The HPBW of the telescope is $18^{\prime \prime} .6(0.04 \mathrm{pc}$ at $450 \mathrm{pc})$ at the frequency used. The total field of view (FoV) for LABOCA is 11!.4. The telescope focus and pointing were checked using the planet Mars and the quasar J0423-013. The submm zenith opacity was determined using the sky-dip method and the values varied from 0.16 to 0.20 , with a median value of 0.18 . The uncertainty due to flux calibration was estimated to be $\sim 10 \%$.

The observations were completed using the on-the-fly (OTF) mapping mode with a scanning speed of $3^{\prime} \mathrm{s}^{-1}$. A single map consisted of 200 scans of $30^{\prime}$ in length in right ascension and spaced by $6^{\prime \prime}$ in declination. The area was observed three times, with a final sensitivity of about $0.03 \mathrm{Jy} \mathrm{beam}^{-1}\left(0.1 M_{\odot}\right.$ beam $^{-1}$ assuming a dust temperature of $10 \mathrm{~K}$ ).

The data reduction was performed using the BoA (BOlometer Array Analysis Software) software package according to guidelines in the BoA User and Reference Manual $(2007)^{4}$. This included flat-fielding, flagging bad/dark channels and data according to telescope speed and acceleration, correcting for the atmospheric opacity, division into subscans, baseline subtractions and median-noise removal, despiking, and filteringout of the low frequencies of the $1 / f$-noise. Finally, the three individual maps were coadded.

\subsection{Spitzer/MIPS archival data}

Pipeline (version S16.1.0)-reduced "post-BCD (Basic Calibrated Data)" Spitzer/MIPS images at 24 and $70 \mu \mathrm{m}$ were downloaded from the Spitzer data archive using the Leopard software package ${ }^{5}$.

We used the software package MOPEX (MOsaicker and Point source EXtractor $)^{6}$ to perform aperture and point-spread function (PSF) fitted photometry on the sources. The point sources were extracted using the APEX package (distributed as part of MOPEX).

At $24 \mu \mathrm{m}$, a 5.31 pixel aperture with a sky annulus of between 8.16 and 13.06 pixels for background subtraction was used. At $70 \mu \mathrm{m}$, the pixel aperture was 8.75 pixels and the sky annulus ranged from 9.75 to 16.25 pixels. The pixel scale

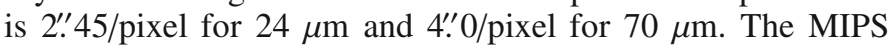

\footnotetext{
${ }^{4}$ http://www . astro.uni-bonn.de/boawiki/Boa

5 http://ssc.spitzer.caltech.edu/propkit/spot/

${ }^{6}$ http://ssc.spitzer. caltech.edu/postbcd/mopex.html
}

resolution is $\sim 6^{\prime \prime}$ and $\sim 18^{\prime \prime}$ at 24 and $70 \mu \mathrm{m}$, respectively. These values correspond to $0.01 \mathrm{pc}$ and $0.04 \mathrm{pc}$, respectively, at the cloud distance of $450 \mathrm{pc}$. The aperture-correction coefficients used with these settings are 1.167 and 1.211 at 24 and $70 \mu \mathrm{m}$, respectively, as given at the Spitzer Science Center (SSC) website $^{7}$. The absolute calibration uncertainties are about $4 \%$ for $24 \mu \mathrm{m}$, and about 10\% for $70 \mu \mathrm{m}$ (Engelbracht et al. 2007; Gordon et al. 2007).

\section{Observational results}

\subsection{Dust emission}

The obtained LABOCA map is presented in Fig. 1. Altogether, 12 compact sources could be identified on this map. A source was deemed real if it had a peak flux density $>5 \sigma$ (i.e., $>0.15 \mathrm{Jy} \mathrm{beam}^{-1}$ ) relative to the local background. The coordinates, peak and integrated flux densities, deconvolved angular FWHM diameters, and axis ratios of the detected sources are listed in Table 2. The coordinates listed relate to the dustemission peaks. The integrated flux densities were derived by summing pixel by pixel the flux density in the source area. The uncertainty in the flux density was derived from $\sqrt{\sigma_{\text {cal }}^{2}+\sigma_{\mathrm{S}}^{2}}$, where $\sigma_{\text {cal }}$ is the uncertainty in the calibration, i.e., $\sim 10 \%$ of flux density, and $\sigma_{\mathrm{S}}$ is the uncertainty in the flux-density determination based on the rms noise level near the source area. We computed the deconvolved source angular diameters, $\theta_{\mathrm{s}}$, assuming that the brightness distribution is Gaussian. The values of $\theta_{\mathrm{s}}$ correspond to the geometric mean of the major and minor axis $F W H M$ obtained from two-dimensional Gaussian fits to the observed emission, which was corrected for the beam size. The uncertainty in $\theta_{\mathrm{s}}$ was calculated by propagating the uncertainties in the major and minor axis $F W H M$, which are formal errors from the Gaussian fit. The axis ratio was defined to be the ratio of deconvolved major axis $F W H M$ to minor axis FWHM. Both the flux-density determination and Gaussian fitting to the sources were completed using the Miriad software package (Sault et al. 1995).

Four of the detected sources have IRAS (Infrared Astronomical Satellite) point-source counterparts, whereas eight are new submm sources. We designated the eight new sources as e.g., SMM 1, SMM 2. The locations of the three $\mathrm{N}_{2} \mathrm{H}^{+}(1-0)$ line emission peaks from Caselli \& Myers (1994) are indicated on the map with plus signs (see Figs. 1 and 3). The $\mathrm{N}_{2} \mathrm{H}^{+}$ peak Ori B9 E, which lies 40" east of IRAS 05405-0117, does not correspond to any submm peak (see Fig. 3). The $\mathrm{N}_{2} \mathrm{H}^{+}$ peak Ori B9 $\mathrm{N}$ lies about 39" southeast of the closest dustcontinuum peak (see Sect. 5.5). One can see that our pointed $\mathrm{N}_{2} \mathrm{H}^{+} / \mathrm{N}_{2} \mathrm{D}^{+}$observations, completed before the LABOCA mapping, missed the strongest submm peak SMM 4 located near IRAS 05405-0117.

\subsection{Spitzer/MIPS images}

The retrieved Spitzer/MIPS images at 24 and $70 \mu \mathrm{m}$ are presented in Fig. 2. All four IRAS sources that were detected by LABOCA are visible at both 24 and $70 \mu \mathrm{m}$ (IRAS 05412-0105 and 05413-0104 northeast of the central region are outside the regions shown in Fig. 2). Of the new submm sources, SMM 3 and SMM 4 are also visible at both 24 and $70 \mu \mathrm{m}$, while there is a $24 \mu \mathrm{m}$ source near SMM 5, which is not detected at $70 \mu \mathrm{m}$. The

\footnotetext{
${ }^{7}$ http://ssc.spitzer.caltech.edu/mips/apercorr
} 


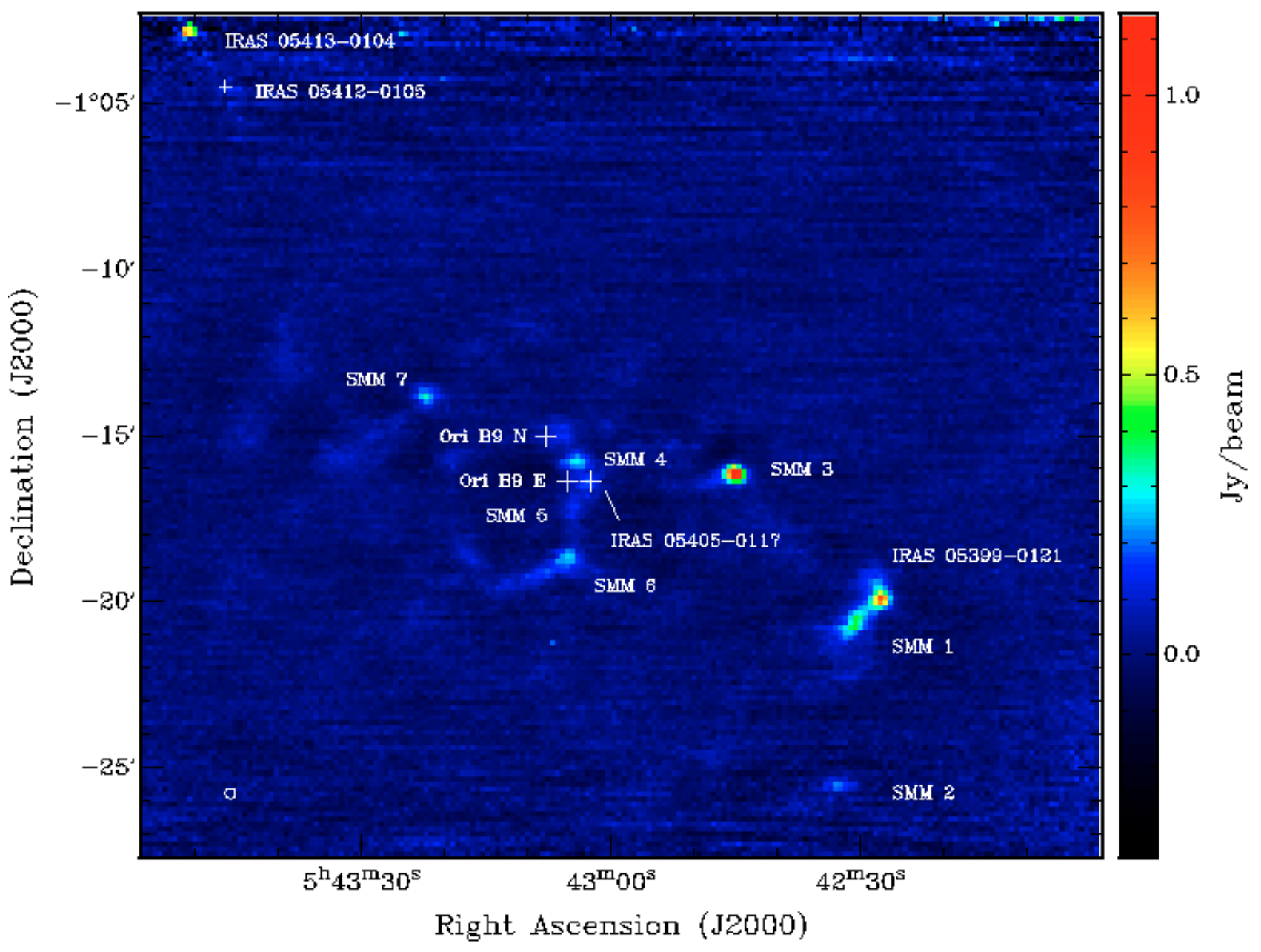

Fig. 1. LABOCA $870 \mu \mathrm{m}$ map of the Ori B9 cloud. The three large plus signs in the centre of the field mark the positions of our molecular-line observations (see Fig. 3). The small plus sign in the upper left shows the dust peak position of IRAS 05412-0105. The beam HPBW (18.'6) is shown in the bottom left.

Table 2. Submillimetre sources in the Ori B9 cloud.

\begin{tabular}{|c|c|c|c|c|c|c|}
\hline \multirow[b]{2}{*}{ Name } & \multicolumn{2}{|c|}{ Peak position } & \multirow{2}{*}{$\begin{array}{c}S_{870}^{\text {peak }} \\
{\left[\mathrm{Jy} \mathrm{beam}^{-1} \text { ] }\right.}\end{array}$} & \multirow{2}{*}{$\begin{array}{l}S_{870} \\
{[\mathrm{Jy}]}\end{array}$} & \multirow{2}{*}{$\begin{array}{c}\theta_{\mathrm{s}} \\
{\left[{ }^{\prime \prime}\right]}\end{array}$} & \multirow[b]{2}{*}{ Axis ratio } \\
\hline & $\alpha_{2000.0}[\mathrm{~h}: \mathrm{m}: \mathrm{s}]$ & $\delta_{2000.0}\left[{ }^{0}:^{\prime}:{ }^{\prime \prime}\right]$ & & & & \\
\hline IRAS 05399-0121 & 054227.4 & -011950 & 0.81 & $2.7 \pm 0.3$ & $30 \pm 4$ & 1.3 \\
\hline SMM 1 & 054230.5 & -012045 & 0.41 & $3.0 \pm 0.3$ & $57 \pm 7$ & 2.5 \\
\hline SMM 2 & 054232.9 & -012528 & 0.21 & $0.7 \pm 0.1$ & $26 \pm 5$ & 3.8 \\
\hline SMM 3 & 054244.4 & -011603 & 1.14 & $2.5 \pm 0.4$ & $19 \pm 3$ & 1.5 \\
\hline IRAS 05405-0117 & 054302.7 & -011621 & 0.19 & $0.9 \pm 0.1^{a}$ & $45 \pm 8^{a}$ & 1.2 \\
\hline SMM 4 & 054303.9 & -011544 & 0.28 & $1.3 \pm 0.1$ & $34 \pm 6$ & 2.3 \\
\hline SMM 5 & 054304.5 & -011706 & 0.16 & $0.6 \pm 0.1$ & $38 \pm 4$ & 1.8 \\
\hline SMM 6 & 054305.1 & -011838 & 0.26 & $2.5 \pm 0.3$ & $92 \pm 35$ & 4.1 \\
\hline Ori B9 N & 054305.7 & -011441 & 0.16 & $1.0 \pm 0.1$ & $47 \pm 5$ & 1.2 \\
\hline SMM 7 & 054322.1 & -011346 & 0.32 & $0.8 \pm 0.1$ & $24 \pm 4$ & 1.8 \\
\hline IRAS 05412-0105 & 054346.4 & -010430 & 0.17 & $0.5 \pm 0.1$ & - & - \\
\hline IRAS 05413-0104 & 054351.3 & -010250 & 0.66 & $0.9 \pm 0.2$ & $25 \pm 8$ & 1.6 \\
\hline
\end{tabular}

${ }^{a}$ These values include both the IRAS 05405-0117 and Ori B9 E (see text and Fig. 3).

remaining submm sources are visible at neither of these wavelength.

In Table 3, we list the sources detected at both 24 and $70 \mu \mathrm{m}$. In this table, we give the 24 and $70 \mu \mathrm{m}$ peak positions of the sources and their flux densities at both wavelengths obtained from the aperture photometry. The $1 \sigma$ uncertainties in the flux densities were derived as described in Sect. 3.1, i.e., as a quadratic sum of the calibration and photometric uncertainties.

\section{3. $\mathrm{N}_{2} \mathrm{H}^{+}$and $\mathrm{N}_{2} \mathrm{D}^{+}$}

The three positions of our molecular-line observations are indicated in Figs. 1 and 3. These positions correspond to the three $\mathrm{N}_{2} \mathrm{H}^{+}(1-0)$ peaks found by Caselli \& Myers (1994; see also Sect. 1.1). The Hanning-smoothed $\mathrm{N}_{2} \mathrm{H}^{+}(1-0)$ and $\mathrm{N}_{2} \mathrm{D}^{+}(2-1)$ spectra are shown in Figs. 4 and 5, respectively. The seven hyperfine components of $\mathrm{N}_{2} \mathrm{H}^{+}(1-0)$ are clearly resolved towards 

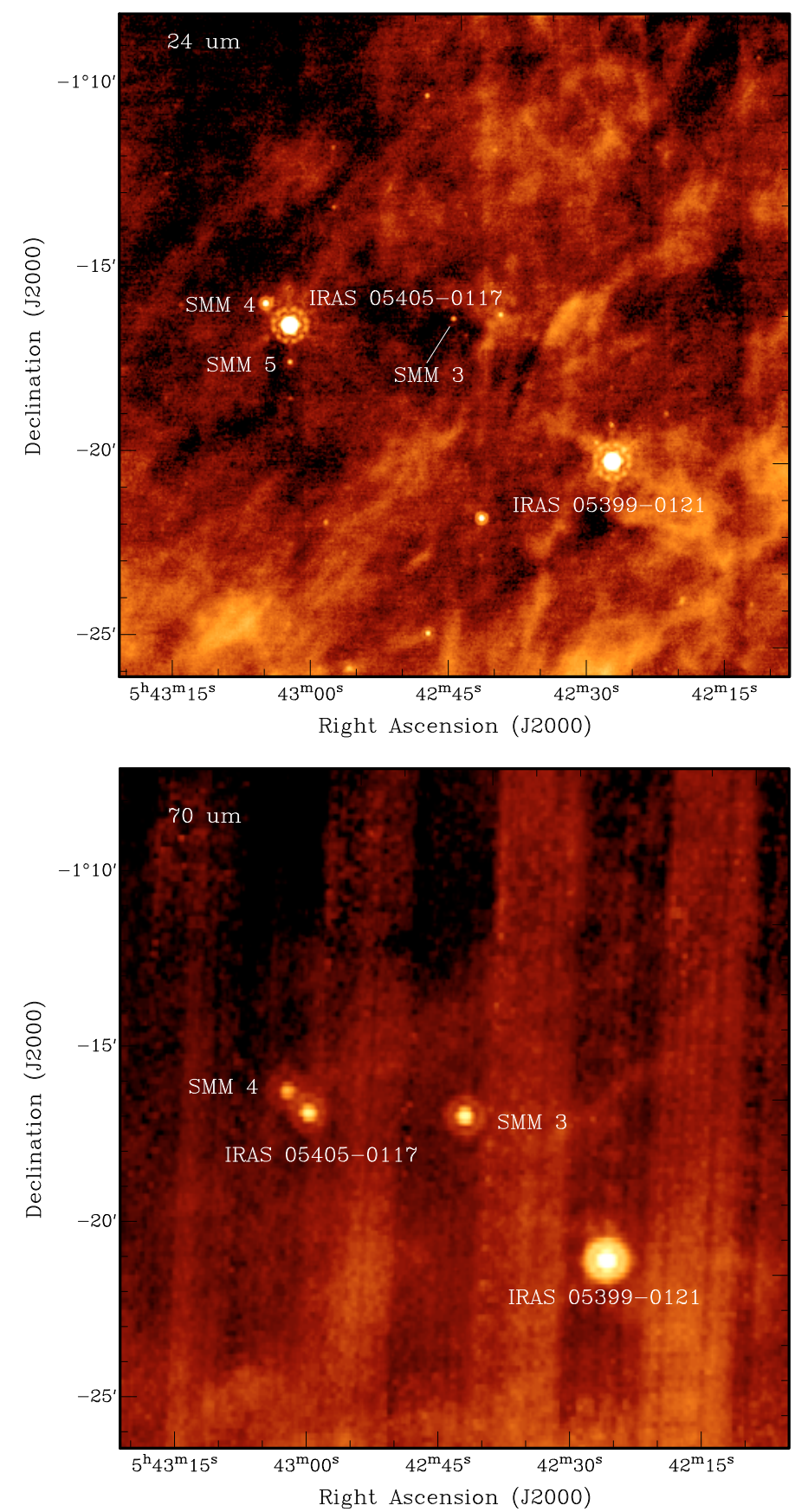

Fig. 2. Spitzer/MIPS $24 \mu \mathrm{m}$ (top) and $70 \mu \mathrm{m}$ (bottom) images of the central part of the Ori B9 cloud. The logarithmic colour scale range from 33.4 to $729.5 \mathrm{MJy} \mathrm{sr}^{-1}$ and 27.9 to $1357.4 \mathrm{MJy} \mathrm{sr}^{-1}$ in the 24 and $70 \mu \mathrm{m}$ images, respectively.

all three positions. The $\mathrm{N}_{2} \mathrm{H}^{+}(1-0)$ spectra towards Ori B9 E and Ori $\mathrm{B} 9 \mathrm{~N}$ show additional lines, which can be explained by $\mathrm{N}_{2} \mathrm{H}^{+}(1-0)$ emission originating in sources of different radial velocity (see Fig. 4). In the case of Ori B9 E, the additional $\mathrm{N}_{2} \mathrm{H}^{+}(1-0)$ lines originate in gas at a radial velocity of $1.3 \mathrm{~km} \mathrm{~s}^{-1}$, whereas towards Ori B9 $\mathrm{N}$, the additional gas component has a $v_{\mathrm{LSR}}$ of $2.2 \mathrm{~km} \mathrm{~s}^{-1}$. These velocities are $\sim 7-8 \mathrm{~km} \mathrm{~s}^{-1}$ lower than the average velocity of the Ori B9 cloud, suggesting that they are produced by a totally different gas component. We checked that the additional components are not caused by, e.g., a phase-lock failure by summing randomly selected subsets of the spectra. All sums constructed in this manner exhibited

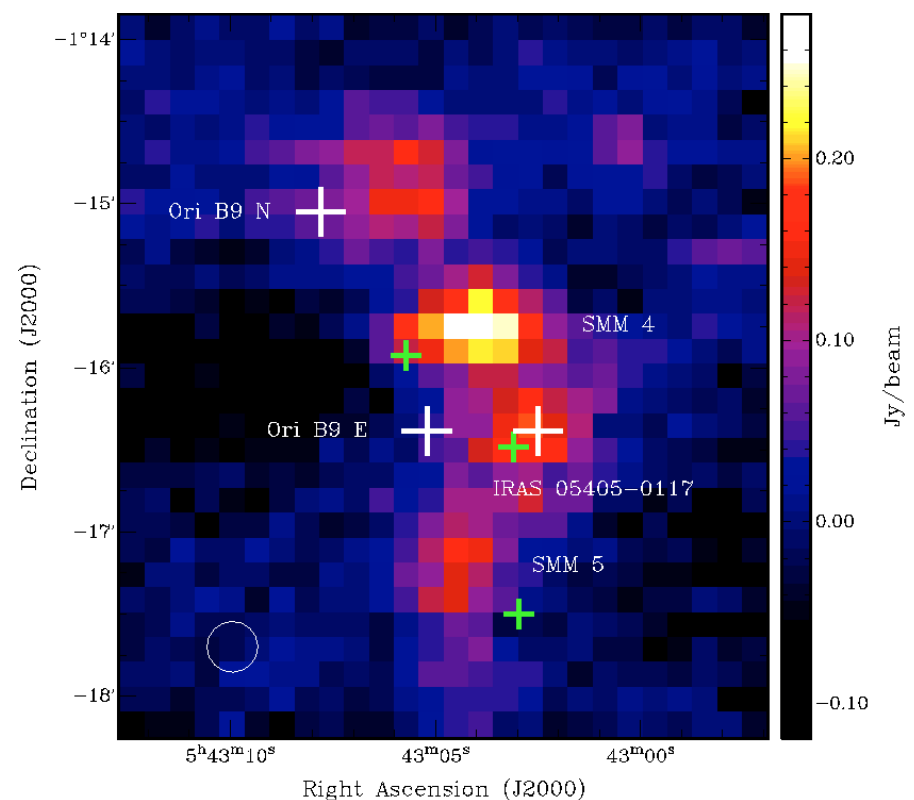

Fig. 3. Zoomed version of Fig. 1 showing the IRAS 05405-0117 clump region. The large plus signs indicate the positions of our $\mathrm{H}_{2} \mathrm{D}^{+}, \mathrm{N}_{2} \mathrm{H}^{+}$, and $\mathrm{N}_{2} \mathrm{D}^{+}$observations towards three condensations shown in Fig. 2 of Caselli \& Myers (1994). Also shown are the $24 \mu \mathrm{m}$ peak positions of SMM 4 and IRAS 05405-0117, and the $24 \mu$ m peak near SMM 5 (small green plus signs, cf. Fig. 2). The beam HPBW (18.'6) is shown in the bottom left.

identical features with equal intensity ratios. The "absorption"like feature at $\sim 20 \mathrm{~km} \mathrm{~s}^{-1}$ in the $\mathrm{N}_{2} \mathrm{H}^{+}(1-0)$ spectrum of Ori $\mathrm{B} 9 \mathrm{~N}$ is an arfefact caused by the frequency-switching folding process.

Only the strongest hyperfine group of $\mathrm{N}_{2} \mathrm{D}^{+}(2-1)$ was detected. The relatively poor signal-to-noise $(\mathrm{S} / \mathrm{N})$ ratio of the data hampers the hyperfine component fitting. Towards Ori B9 N, the additional velocity component at $\sim 2.2 \mathrm{~km} \mathrm{~s}^{-1}$ was also detected in $\mathrm{N}_{2} \mathrm{D}^{+}(2-1)$.

In Table 4, we give the $\mathrm{N}_{2} \mathrm{H}^{+}(1-0)$ and $\mathrm{N}_{2} \mathrm{D}^{+}(2-1)$ line parameters derived from the Hanning-smoothed spectra. The LSR velocities and line-widths (FWHM) are listed in Cols. 2 and 3, respectively. The total optical depth and excitation temperature of the lines are given in Cols. 6 and 7, respectively. The excitation temperatures, $T_{\text {ex }}$, of the $\mathrm{N}_{2} \mathrm{H}^{+}(1-0)$ transition were derived from the antenna equation

$T_{\mathrm{A}}^{*}=\eta \frac{h v}{k_{\mathrm{B}}}\left[F\left(T_{\mathrm{ex}}\right)-F\left(T_{\mathrm{bg}}\right)\right]\left(1-\mathrm{e}^{-\tau}\right)$,

where $\eta$ is the beam-source coupling efficiency, $h$ is the Planck constant, $v$ is the transition frequency, $k_{\mathrm{B}}$ is the Boltzmann constant, $T_{\mathrm{bg}}=2.725 \mathrm{~K}$ is the cosmic microwave background (CMB) temperature, and the function $F(T)$ is defined by $F(T) \equiv$ $\left(\mathrm{e}^{h v / k_{\mathrm{B}} T}-1\right)^{-1}$. We assumed that $\eta=\eta_{\mathrm{MB}}$, and we used the main beam brightness temperature, $T_{\mathrm{MB}}=\eta_{\mathrm{MB}}^{-1} T_{\mathrm{A}}^{*}$, and the optical thickness, $\tau$, of the brightest hyperfine component. The uncertainty in $T_{\mathrm{ex}}$ was calculated by propagating the uncertainties in $T_{\mathrm{MB}}$ and $\tau$.

The total optical depth of the $\mathrm{N}_{2} \mathrm{D}^{+}(2-1)$ line cannot be calculated directly because the hyperfine components are not resolved in the spectra. We estimated the total optical depth in the following manner. First, we calculated the optical depth of the main hyperfine group of $\mathrm{N}_{2} \mathrm{D}^{+}(2-1)$ from the antenna equation using the $T_{\mathrm{MB}}$ obtained from a Gaussian fit to the group 
Table 3. Spitzer 24/70 $\mu \mathrm{m}$ sources in Ori B9.

\begin{tabular}{ccccccc}
\hline \hline & \multicolumn{2}{c}{$24 \mu \mathrm{m}$ peak position } & \multicolumn{2}{c}{$70 \mu \mathrm{m}$ peak position } & $S_{24}$ & $S_{70}$ \\
Name & $\alpha_{2000.0}[\mathrm{~h}: \mathrm{m}: \mathrm{s}]$ & $\delta_{2000.0}\left[{ }^{\circ}::^{\prime \prime}\right]$ & $\alpha_{2000.0}[\mathrm{~h}: \mathrm{m}: \mathrm{s}]$ & $\delta_{2000.0}\left[:^{\circ}::^{\prime \prime}\right]$ & {$[\mathrm{Jy}]$} & {$[\mathrm{Jy}]$} \\
\hline IRAS 05399-0121 & 054227.6 & -012001 & 054227.7 & -011957 & $1.3 \pm 0.05$ & $24.4 \pm 2.4$ \\
SMM 3 & 054245.3 & -011614 & 054245.1 & -011613 & $0.005 \pm 0.0002$ & $3.6 \pm 0.4$ \\
IRAS 05405-0117 & 054303.1 & -011629 & 054303.0 & -011630 & $1.3 \pm 0.05$ & $2.7 \pm 0.3$ \\
SMM 4 & 054305.7 & -011555 & 054305.6 & -011552 & $0.036 \pm 0.001$ & $1.3 \pm 0.1$ \\
IRAS 05412-0105 & 054346.3 & -010444 & 054346.1 & -010443 & $0.6 \pm 0.02$ & $2.5 \pm 0.3$ \\
IRAS 05413-0104 & 054351.4 & -010253 & 054351.3 & -010251 & $0.2 \pm 0.01$ & $17.9 \pm 1.8$ \\
\hline
\end{tabular}
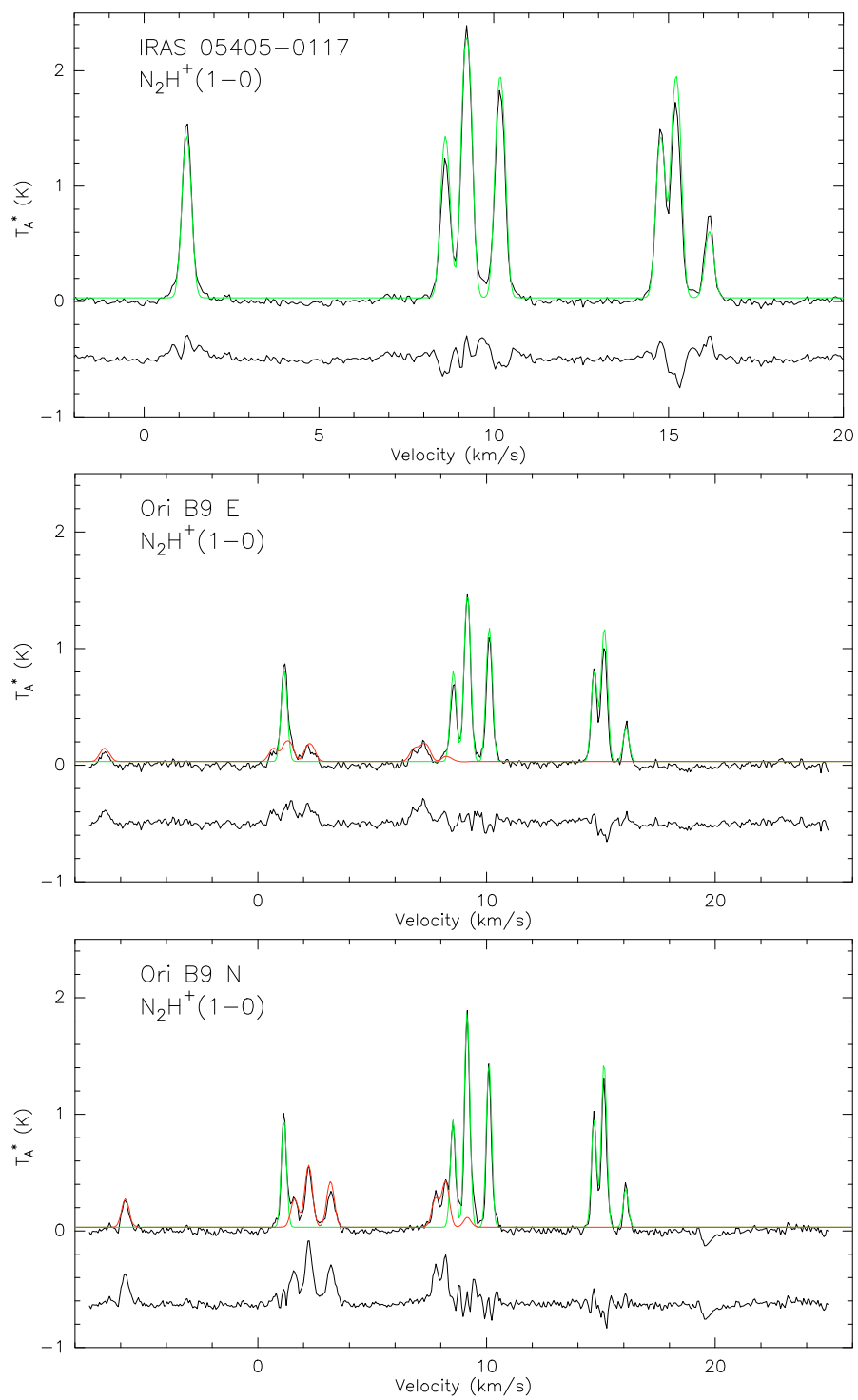

Fig. 4. $\mathrm{N}_{2} \mathrm{H}^{+}(1-0)$ spectra toward IRAS 05405-0117 (top), Ori B9 E (middle), and Ori B9 N (bottom) after Hanning smoothing. Hyperfine fits to the spectra are indicated by green lines. The residuals of the fits are shown below the spectra. Hyperfine fits to the other velocity component are indicated by red lines (see text). The small "absorption"-like feature at $\sim 20 \mathrm{~km} \mathrm{~s}^{-1}$ in the bottom panel is an arfefact caused by frequency switching.

of 4 strongest hyperfines. In the calculation, we adopted the excitation temperature of the $\mathrm{N}_{2} \mathrm{H}^{+}(1-0)$ lines. Second, the total optical depths of $\mathrm{N}_{2} \mathrm{D}^{+}(2-1)$ were calculated after taking into account that the main group corresponds to $54.3 \%$ of $\tau_{\text {tot }}$. The uncertainty in $\tau_{\text {tot }}$ was calculated by propagating the uncertainties in $T_{\mathrm{MB}}$ and $T_{\mathrm{ex}}$.
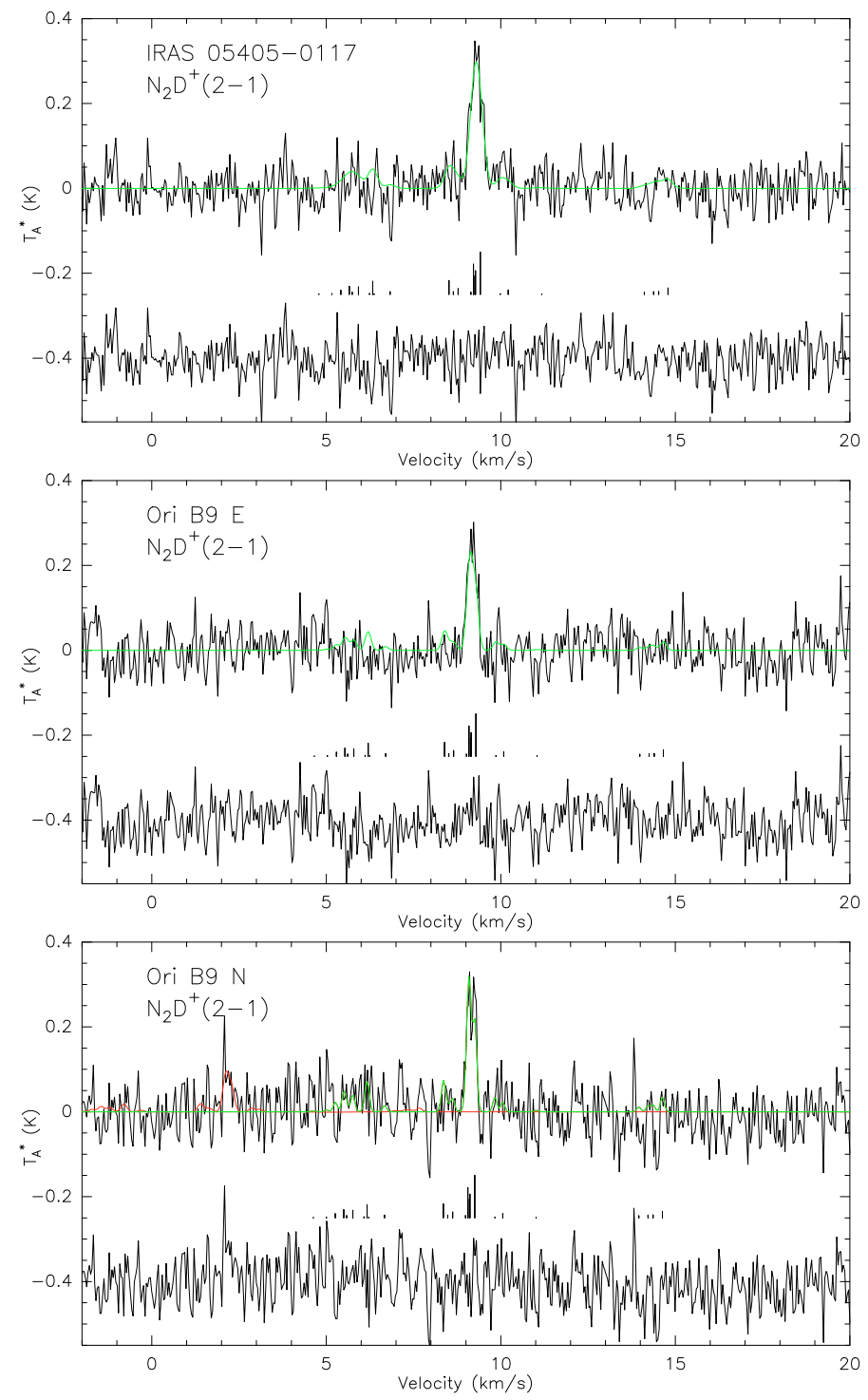

Fig. 5. $\mathrm{N}_{2} \mathrm{D}^{+}(2-1)$ spectra toward IRAS 05405-0117 (top), Ori B9 E (middle), and Ori B9 N (bottom) after Hanning smoothing. Hyperfine fits to the spectra are indicated by green lines. The lines under the spectra indicate the positions and relative intensities of the hyperfine components (see Table 2 in Gerin et al. 2001). Undermost are plotted the residuals of the fits. A hyperfine fit to the other velocity component in the bottom panel is indicated by a red line (see text).

\section{Physical and chemical parameters of the sources}

In this section, we outline the methods used to derive the physical and chemical parameters of the sources, and present the results obtained. 
Table 4. $\mathrm{N}_{2} \mathrm{H}^{+}(1-0)$ and $\mathrm{N}_{2} \mathrm{D}^{+}(2-1)$ line parameters derived from Hanning smoothed spectra.

\begin{tabular}{ccccccc}
\hline \hline Line/Position & $v_{\text {LSR }}\left[\mathrm{km} \mathrm{s}^{-1}\right]$ & $\Delta v\left[\mathrm{~km} \mathrm{~s}^{-1}\right]$ & $T_{\mathrm{A}}^{*}[\mathrm{~K}]$ & $\int T_{\mathrm{A}}^{*}(v) \mathrm{d} v\left[\mathrm{~K} \mathrm{~km} \mathrm{~s}^{-1}\right]$ & $\tau_{\text {tot }}$ & $T_{\text {ex }}[\mathrm{K}]$ \\
\hline $\mathbf{N}_{2} \mathbf{H}^{+}(1-0)$ & & & & & & \\
IRAS 05405-0117 & $9.228 \pm 0.001$ & $0.290 \pm 0.002$ & $2.37 \pm 0.04$ & $3.97 \pm 0.05$ & $6.1 \pm 0.03$ & $6.8 \pm 0.07$ \\
Ori B9 E & $9.163 \pm 0.002$ & $0.298 \pm 0.005$ & $1.46 \pm 0.04$ & $2.26 \pm 0.03$ & $3.5 \pm 0.5$ & $6.1 \pm 0.3$ \\
Ori B9 N & $9.149 \pm 0.003$ & $0.261 \pm 0.008$ & $1.82 \pm 0.09$ & $2.29 \pm 0.04$ & $2.2 \pm 0.8$ & $8.3 \pm 1.4$ \\
$\mathbf{N}_{2} \mathbf{D}^{+}(2-1)$ & & & & & & \\
IRAS 05405-0117 & $9.414 \pm 0.012$ & $0.319 \pm 0.027$ & $0.31 \pm 0.04$ & $0.13 \pm 0.01$ & $0.26 \pm 0.01^{d}$ & $6.8 \pm 0.07$ \\
Ori B9 E & $9.285 \pm 0.013$ & $0.194 \pm 0.029$ & $0.27 \pm 0.03$ & $0.09 \pm 0.01$ & $0.28 \pm 0.03^{d}$ & $6.1 \pm 0.3$ \\
Ori B9 N & $9.255 \pm 0.009$ & $0.136 \pm 0.021$ & $0.28 \pm 0.07$ & $0.09 \pm 0.01$ & $0.16 \pm 0.05^{d}$ & $8.3 \pm 1.4$ \\
\hline
\end{tabular}

The integrated line intensity $\left(\int T_{\mathrm{A}}^{*}(v) \mathrm{d} v\right)$ includes all the hyperfine components in the case of $\mathrm{N}_{2} \mathrm{H}^{+}$, whereas for $\mathrm{N}_{2} \mathrm{D}^{+}$only the main group is included.

${ }^{a}$ Caselli \& Myers (1994) derived $v_{\mathrm{LSR}}=9.209 \pm 0.003 \mathrm{~km} \mathrm{~s}^{-1}, \Delta v=0.313 \pm 0.008 \mathrm{~km} \mathrm{~s}^{-1}$, and $\tau_{\text {tot }}=4.594 \pm 0.825 .{ }^{b}$ For the other velocity component hyperfine fit yields $v_{\mathrm{LSR}}=1.310 \pm 0.013 \mathrm{~km} \mathrm{~s}^{-1}, \Delta v=0.436 \pm 0.035 \mathrm{~km} \mathrm{~s}^{-1}$, and $\tau_{\text {tot }}=6.6 \pm 2.0 .^{c}$ For the other velocity component $v_{\text {LSR }}=2.219 \pm 0.006 \mathrm{~km} \mathrm{~s}^{-1}, \Delta v=0.378 \pm 0.021 \mathrm{~km} \mathrm{~s}^{-1}, T_{\mathrm{A}}^{*}=0.52 \pm 0.03 \mathrm{~K}, \tau_{\text {tot }}=1.0$, and $T_{\mathrm{ex}}=5.9 \pm 0.17 \mathrm{~K} .{ }^{d} \tau_{\text {tot }}$ calculated by taking into account that the main hyperfine group corresponds to $54.3 \%$ of the total optical depth. $\tau_{\text {main group }}$ is calculated using $T_{\mathrm{MB}}$ from Gaussian fit to the main group and $T_{\mathrm{ex}}$ from $\mathrm{N}_{2} \mathrm{H}^{+}(1-0) .{ }^{e}$ For the other velocity component $v_{\mathrm{LSR}}=2.290 \pm 0.044 \mathrm{~km} \mathrm{~s}^{-1}, \Delta v=0.187 \pm 0.090 \mathrm{~km} \mathrm{~s}^{-1}$, $T_{\mathrm{A}}^{*}=0.22 \pm 0.06 \mathrm{~K}$, and $\tau_{\text {tot }}=0.12 \pm 0.01\left(\tau_{\text {tot }}\right.$ is calculated as described in footnote $\left.d\right)$.

Table 5. IRAS flux densities in Jy.

\begin{tabular}{ccccc}
\hline \hline Name & $S_{12}$ & $S_{25}$ & $S_{60}$ & $S_{100}$ \\
\hline IRAS 05399-0121 & 0.25 & 1.59 & 22.94 & 45.93 \\
IRAS 05405-0117 & 0.40 & 1.55 & 3.75 & 19.67 \\
IRAS 05412-0105 & 0.26 & 0.65 & 1.66 & 73.58 \\
IRAS 05413-0104 & 0.25 & 0.31 & 17.33 & 59.46 \\
\hline
\end{tabular}

\subsection{Spectral energy distributions}

The 24 and $70 \mu \mathrm{m}$ flux densities and the integrated flux densities at $870 \mu \mathrm{m}$ were used to fit the spectral energy distribution (SED) of SMM 3 and SMM 4. For the IRAS sources, the archival IRAS data were also included. The flux densities in the 12, 25, 60, and $100 \mu \mathrm{m}$ IRAS bands are listed in Table 5. The derived SEDs for SMM 3, SMM 4, and IRAS 05405-0117 are shown in Fig. 6.

For all six sources detected at three or more wavelengths, the data were fitted by a two-temperature composite model. The parameters resulting from the fitting are given in Table 6. We adopted a gas-to-dust mass ratio of 100, and dust opacities corresponding to a MRN size distribution with thick ice mantles at a gas density of $n_{\mathrm{H}}=10^{5} \mathrm{~cm}^{-3}$ (Ossenkopf \& Henning 1994). The total (cold+warm) mass and the integrated bolometric luminosity are given in Cols. 2 and 3 of Table 6, respectively. The temperatures of the two components are listed in Cols. 4 and 5. In Cols. 6 and 7, we indicate the mass and luminosity fractions of the cold component versus the total mass and luminosity, and in Col. 8, we list the ratio of submm luminosity (numerically integrated longward of $350 \mu \mathrm{m}$ ) to total bolometric luminosity $\left(L_{\text {submm }} / L_{\text {bol }}\right)$. Column 9 lists the normalised envelope mass, $M_{\text {tot }} / L_{\text {bol }}^{0.6}$, which is an evolutionary indicator because it correlates with the protostellar outflow strength (i.e., with the mass-accretion rate), and thus decreases with time (Bontemps et al. 1996). In Col. 10, we indicate the source SED classification (see Sect. 5.1). In all cases, the mass of the warm component is negligible $\left(\sim 10^{-7}-10^{-4} M_{\odot}\right)$ and thus the bulk of the material is cold $\left(M_{\mathrm{cold}} / M_{\mathrm{tot}} \sim 1\right)$.

\subsection{Linear sizes, and mass estimates and densities}

The linear sizes (radii $R=\theta_{\mathrm{s}} d / 2$ ) were computed from the angular FWHM sizes listed in Table 2. The masses of the cores (gas+dust mass, $M_{\text {cont }}$ ) were calculated from their integrated $870 \mu \mathrm{m}$ continuum flux density, $S_{870}$, assuming that the thermal dust emission is optically thin:

$M_{\text {cont }}=\frac{S_{870} d^{2}}{B_{870}\left(T_{\mathrm{d}}\right) \kappa_{870} R_{\mathrm{d}}}$,

where $d$ is the distance, and $B_{870}\left(T_{\mathrm{d}}\right)$ is the Planck function with dust temperature $T_{\mathrm{d}}$. For all the IRAS sources as well as for SMM 3 and SMM 4, we adopted the dust temperatures resulting from the SED fitting (see Table 6, Col. 4). For all the other sources, it was assumed that $T_{\mathrm{d}}=10 \mathrm{~K}$. The assumed dust temperature of $10 \mathrm{~K}$ is justified by estimates derived from $\mathrm{NH}_{3}$ (Harju et al. 1993) and is commonly adopted for starless cores. The assumption that $T_{\mathrm{d}}=T_{\text {kin }}$, where $T_{\text {kin }}$ is the gas kinetic temperature, is probably valid at densities $n\left(\mathrm{H}_{2}\right)>10^{5} \mathrm{~cm}^{-3}$ (Burke \& Hollenbach 1983). The opacity per unit mass column density at $\lambda=870 \mu \mathrm{m}$ is assumed to be $\kappa_{870} \simeq 0.17 \mathrm{~m}^{2} \mathrm{~kg}^{-1}$. This value is interpolated from Ossenkopf \& Henning (1994, see Sect. 4.1). The value $1 / 100$ is adopted for the dust-to-gas mass ratio, $R_{\mathrm{d}}$.

The virial masses, $M_{\mathrm{vir}}$, of IRAS 05405-0117 and Ori B9 N were estimated by approximating the mass distribution by a homogenous, isothermal sphere without magnetic support and external pressure (see, e.g., Eqs. (1) and (2) in Chen et al. 2008, where the line-width of $\mathrm{N}_{2} \mathrm{H}^{+}$is used). The resulting virial masses are about 4.3 $M_{\odot}$ for IRAS 05405-0117 and 2.8 $M_{\odot}$ for Ori B9 N. The corresponding $M_{\text {cont }} / M_{\text {vir }}$ ratios are about 0.3 and 1.4. We note that it is usual for protostellar cores, such as IRAS 05405-0117, to appear below the self-gravitating limit $\left(M_{\text {cont }} / M_{\text {vir }}=0.5\right)$, though they are forming stars (e.g., Enoch et al. 2008). Since Ori B9 N appears to be gravitationally bound, it is probably prestellar. There are several factors that would lead to virial masses being overestimated. For example, using the radial density profile with power-law indices $p=1-1.5$ (see Sect. 5.3) would reduce $M_{\text {vir }}$ by factors of 1.1-1.25.

The volume-averaged $\mathrm{H}_{2}$ number densities, $\left\langle n\left(\mathrm{H}_{2}\right)\right\rangle$, were calculated assuming a spherical geometry for the sources and using masses, $M_{\text {cont }}$, and radii, $R$, estimated for them from the dust continuum map. The obtained radii, masses, and volumeaveraged $\mathrm{H}_{2}$ number densities are given in Cols. 2, 3, and 5 of Table 7 , respectively. 

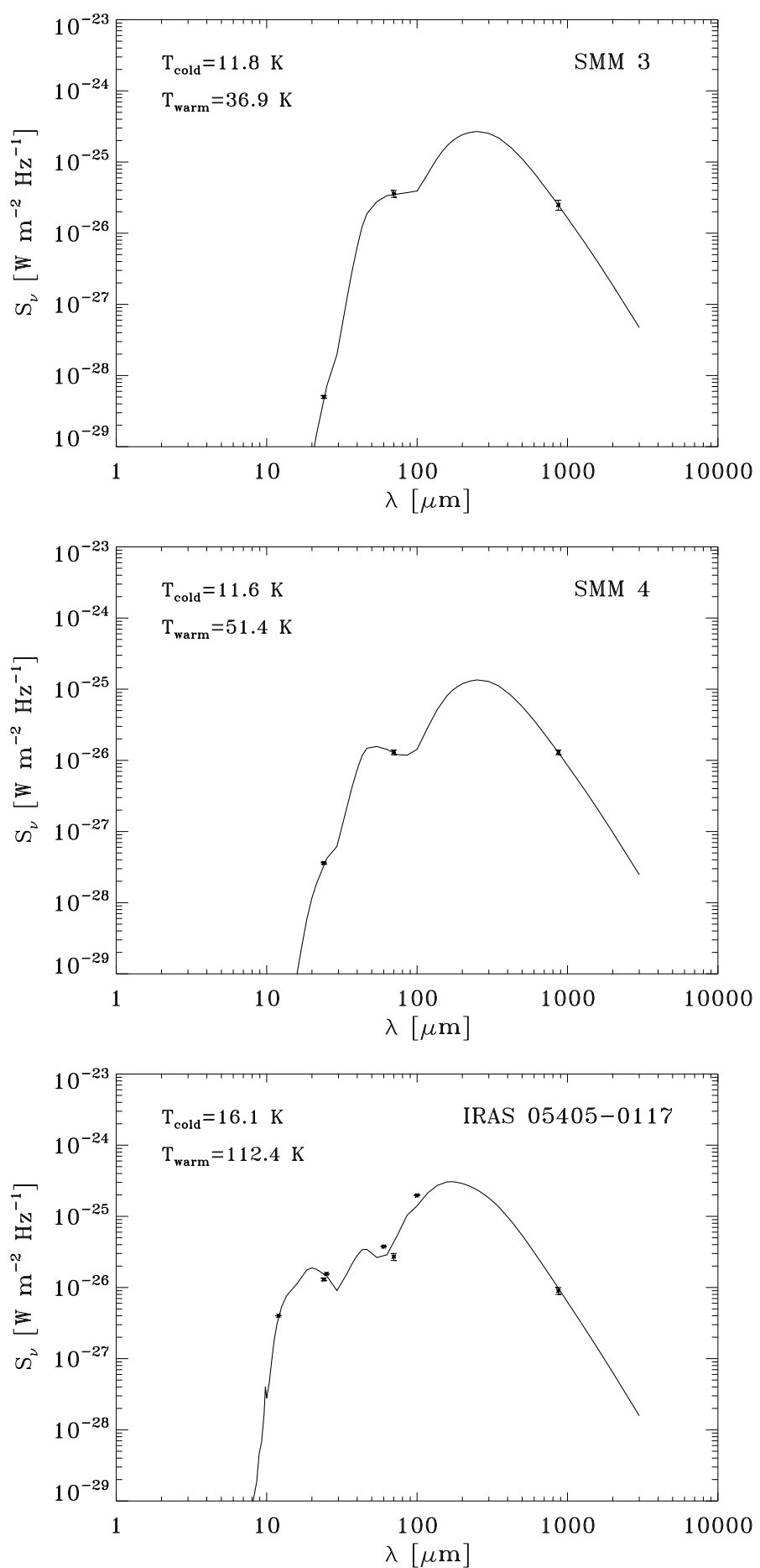

Fig. 6. SEDs of the sources SMM 3 (top), SMM 4 (middle), and IRAS 05045-0117 (bottom). 24 and $70 \mu \mathrm{m}$ data points are derived from archival Spitzer/MIPS data, while the $870 \mu \mathrm{m}$ measurement is performed with LABOCA. For IRAS 05045-0117 we include also IRAS archival data (see Table 5). $1 \sigma$ error bars are indicated for Spitzer and LABOCA data points. The solid lines in all plots represent the sum of two (cold+warm) components (see Cols. (4) and (5) of Table 6). The "ripple" between $\sim 30-60 \mu \mathrm{m}$ in the SED of IRAS 05045-0117 is due to simple logarithmic interpolation used to derive the luminosity.

\subsubsection{Total mass of the Ori B9 region}

We estimated the total mass in the region by using the nearinfrared extinction mapping technique (NICER, Lombardi \& Alves 2001). In this technique, the near-infrared colours, namely
$H-K$ and $J-H$, of the stars shining through the dust cloud are compared to the colours of stars in a nearby field that is free from dust. The reddened colours of the stars behind the dust cloud can then be interpreted in terms of extinction caused by the relatively well-known ratios of optical depths at JHK wavelengths (for further details of the method, we refer to Lombardi \& Alves 2001). To implement the method, we retrieved $J H K$ photometric data from the 2MASS archive, covering a $30^{\prime} \times 19^{\prime}$ region centred on $(\alpha, \delta)_{\mathrm{J} 2000}=(5: 43: 00,-01: 16: 20)$. Applying NICER to these data yielded an extinction map of resolution $F W H M=2.5$, indicating extinction values of $A_{\mathrm{V}}=8 \ldots 12 \mathrm{mag}$ at the positions of the detected sources.

The total mass of the region was calculated from the derived extinction map by summing the extinction values of all pixels assuming the gas-to-dust ratio of $N(\mathrm{H})=2 \times$ $10^{21} \mathrm{~cm}^{-2} \mathrm{mag}^{-1}$ (Bohlin et al. 1978), and the mean molecular weight per $\mathrm{H}_{2}$ molecule of 2.8. The total mass of the region inferred from this calculation was $1400 M_{\odot}$. The total mass of the cores within the region implied by the submm dust emission data is only $\sim 50 M_{\odot}$, about $3.6 \%$ of the total mass in the region.

\subsection{Column densities, fractional abundances, and the degree of deuterium fractionation}

The $\mathrm{H}_{2}$ column densities, $N\left(\mathrm{H}_{2}\right)$, towards the submm peaks and the positions selected for the line observations were calculated using the equation

$N\left(\mathrm{H}_{2}\right)=\frac{I_{870}^{\text {dust }}}{B_{870}\left(T_{\mathrm{d}}\right) \mu_{\mathrm{H}_{2}} m_{\mathrm{H}} K_{870} R_{\mathrm{d}}}$,

where $I_{870}^{\text {dust }}$ is the observed dust peak surface brightness, which is related to the peak flux density via $1 \mathrm{Jy} / 18^{\prime \prime}$. 6 beam $=1.085 \times$ $10^{-18} \mathrm{~W} \mathrm{~m}^{-2} \mathrm{~Hz}^{-1} \mathrm{sr}^{-1}, \mu_{\mathrm{H}_{2}}=2.8$ is the mean molecular weight per $\mathrm{H}_{2}$ molecule, and $m_{\mathrm{H}}$ is the mass of the hydrogen atom. The dust temperature values used were identical to those adopted in the mass estimates (Eq. (2)).

The $\mathrm{N}_{2} \mathrm{H}^{+}$column densities were calculated using the equation

$N_{\text {tot }}=\frac{3 \epsilon_{0} h}{2 \pi^{2} \mu_{\mathrm{el}}^{2}} \frac{1}{S_{\mathrm{ul}}} \mathrm{e}^{E_{\mathrm{u}} / k_{\mathrm{B}} T_{\mathrm{ex}}} F\left(T_{\mathrm{ex}}\right) Z\left(T_{\mathrm{ex}}\right) \int \tau(v) \mathrm{d} v$,

where $\epsilon_{0}$ is the vacuum permittivity, $\mu_{\mathrm{el}}$ is the permanent electric dipole moment, $S_{\mathrm{ul}}$ is the line strength, $E_{\mathrm{u}}$ is the upper state energy, $Z$ is the rotational partition function, and $\int \tau \mathrm{d} v$ is the integrated optical thickness. We assumed a dipole moment of 3.4 D for both $\mathrm{N}_{2} \mathrm{H}^{+}$and $\mathrm{N}_{2} \mathrm{D}^{+}$(Havenith et al. 1990). For the rotational transition $J_{\mathrm{u}} \rightarrow J_{\mathrm{u}}-1$ of a linear molecule (like $\mathrm{N}_{2} \mathrm{H}^{+}$), $S_{\mathrm{ul}}=J_{\mathrm{u}}$.

For the $\mathrm{N}_{2} \mathrm{H}^{+}$lines, the optical thicknesses were derived from the Gaussian fits to the hyperfine components, and thus the integral $\int \tau \mathrm{d} v$ can be replaced by $\frac{\sqrt{\pi}}{2 \sqrt{\ln 2}} \Delta v \tau_{0}$. Here $\Delta v$ is the linewidth of an individual hyperfine component, and $\tau_{0}$ is the sum of the peak optical thicknesses of all the seven components.

The $\mathrm{N}_{2} \mathrm{D}^{+}$column densities were calculated in two different ways: 1) as in the case of $\mathrm{N}_{2} \mathrm{H}^{+}$, and 2) using Eq. (1) with the approximation of an optically thin line $(\tau \ll 1)$ :

$T_{\mathrm{A}}^{*} \approx \eta \frac{h v}{k_{\mathrm{B}}}\left[F\left(T_{\mathrm{ex}}\right)-F\left(T_{\mathrm{bg}}\right)\right] \tau$, 
Table 6. Results of the SED fits.

\begin{tabular}{|c|c|c|c|c|c|c|c|c|c|}
\hline Source & $\begin{array}{l}M_{\text {tot }} \\
{\left[M_{\odot}\right]}\end{array}$ & $\begin{array}{l}L_{\text {bol }} \\
{\left[L_{\odot}\right]}\end{array}$ & $\begin{array}{l}T_{\text {cold }} \\
{[\mathrm{K}]}\end{array}$ & $\begin{array}{c}T_{\text {warm }} \\
{[\mathrm{K}]}\end{array}$ & $M_{\mathrm{cold}} / M_{\mathrm{tot}}$ & $L_{\text {cold }} / L_{\text {bol }}$ & $L_{\text {submm }} / L_{\text {bol }}$ & $\begin{array}{l}M_{\text {tot }} / L_{\text {bol }}^{0.6} \\
{\left[M_{\odot} / L_{\odot}^{0.6}\right]}\end{array}$ & Class \\
\hline IRAS 05399-0121 & $2.8 \pm 0.3$ & $21 \pm 1.2$ & $18.5 \pm 0.1$ & $103.9 \pm 0.2$ & $\sim 1$ & 0.90 & 0.02 & 0.45 & $0 / \mathrm{I}$ \\
\hline SMM 3 & $7.2 \pm 2.2$ & $3.5 \pm 0.2$ & $11.8 \pm 0.9$ & $36.9 \pm 0.2$ & $\sim 1$ & 0.74 & 0.11 & 5.57 & 0 \\
\hline IRAS 05405-0117 & $1.6 \pm 0.2$ & $6.4 \pm 0.4$ & $16.1 \pm 0.1$ & $112.4 \pm 0.4$ & $\sim 1$ & 0.69 & 0.03 & 0.53 & 0 \\
\hline SMM 4 & $3.8 \pm 0.2$ & $1.7 \pm 0.2$ & $11.6 \pm 0.2$ & $51.4 \pm 4.9$ & $\sim 1$ & 0.76 & 0.11 & 2.76 & 0 \\
\hline IRAS 05412-0105 & $1.3 \pm 0.3$ & $5.8 \pm 0.6$ & $17.0 \pm 0.3$ & $127.8 \pm 0.5$ & $\sim 1$ & 0.86 & 0.03 & 0.45 & 0 \\
\hline IRAS 05413-0104 & $1.0 \pm 0.2$ & $13.2 \pm 1.2$ & $20.3 \pm 0.4$ & $152.3 \pm 1.1$ & $\sim 1$ & 0.97 & 0.02 & 0.21 & 0 \\
\hline
\end{tabular}

Table 7. Linear radii, masses, and $\mathrm{H}_{2}$ column and volume-averaged number densities of all detected submm sources.

\begin{tabular}{ccccc}
\hline \hline Source & $\begin{array}{c}R \\
{[\mathrm{pc}]}\end{array}$ & $\begin{array}{c}M_{\text {cont }} \\
{\left[M_{\odot}\right]}\end{array}$ & $\begin{array}{c}N\left(\mathrm{H}_{2}\right) \\
{\left[10^{22} \mathrm{~cm}^{-2}\right]}\end{array}$ & $\begin{array}{c}\left\langle n\left(\mathrm{H}_{2}\right)\right\rangle \\
{\left[10^{5} \mathrm{~cm}^{-3}\right]}\end{array}$ \\
\hline IRAS 05399-0121 & 0.03 & 3.6 & 2.61 & 7.5 \\
SMM 1 & 0.06 & 11.8 & 3.89 & 3.1 \\
SMM 2 & 0.03 & 2.8 & 1.99 & 5.8 \\
SMM 3 & 0.02 & 7.2 & 7.80 & 51.1 \\
IRAS 05405-0117 & $0.05^{a}$ & $1.5^{a}$ & 0.76 & $0.7^{a}$ \\
SMM 4 & 0.04 & 3.8 & 1.98 & 3.4 \\
SMM 5 & 0.04 & 2.3 & 1.51 & 2.1 \\
SMM 6 & 0.10 & 9.9 & 2.45 & 0.6 \\
Ori B9 N & 0.05 & 3.9 & 1.51 & 1.7 \\
SMM 7 & 0.03 & 3.1 & 3.01 & 6.5 \\
IRAS 05412-0105 & $-b$ & 0.8 & 0.63 & $-{ }^{b}$ \\
IRAS 05413-0104 & 0.03 & 1.0 & 1.85 & 2.2 \\
\hline
\end{tabular}

${ }^{a}$ These values include both the IRAS 05405-0117 and Ori B9 E.

${ }^{b}$ Deconvolving the angular size was not possible, and thus the radius and number density could not be estimated.

We again assumed that $\eta=\eta_{\mathrm{MB}}$. The integrated opacity was estimated from the integrated $T_{\mathrm{MB}}$ of the main hyperfine group (54.3\% of the total integrated intensity):

$$
\int \tau \mathrm{d} v=\frac{\int T_{\mathrm{MB}} \mathrm{d} v}{\frac{h v}{k_{\mathrm{B}}}\left[F\left(T_{\mathrm{ex}}\right)-F\left(T_{\mathrm{bg}}\right)\right]} .
$$

A comparison of the column density determined using the two methods shows that the measurement of $N\left(\mathrm{~N}_{2} \mathrm{D}^{+}\right)$derived using the first method, is a factor of 1.3 (IRAS 05405-0117), 0.7 (Ori B9 E), and 0.6 (Ori B9 N) of the value obtained using the second method. Since the $\mathrm{N}_{2} \mathrm{D}^{+}$line areas are quire uncertain, the $\mathrm{N}_{2} \mathrm{D}^{+}$column densities determined by using the first method were adopted in this paper.

The fractional $\mathrm{N}_{2} \mathrm{H}^{+}$and $\mathrm{N}_{2} \mathrm{D}^{+}$abundances, $x\left(\mathrm{~N}_{2} \mathrm{H}^{+}\right)$and $x\left(\mathrm{~N}_{2} \mathrm{D}^{+}\right)$, were calculated by dividing the corresponding column densities by $N\left(\mathrm{H}_{2}\right)$ from the dust continuum. For $x\left(\mathrm{~N}_{2} \mathrm{H}^{+}\right)$, the dust map was smoothed to 26 .' 4 , the resolution of the $\mathrm{N}_{2} \mathrm{H}^{+}$observations. No smoothing was completed in the case of $\mathrm{N}_{2} \mathrm{D}^{+}$, since the resolutions of the $\mathrm{N}_{2} \mathrm{D}^{+}$and dust continuum observations were similar (16.' 0 and 18 .' $^{\prime} 6$, respectively). The degree of deuterium fractionation in $\mathrm{N}_{2} \mathrm{H}^{+}$is defined as the column density ratio $R_{\text {deut }} \equiv N\left(\mathrm{~N}_{2} \mathrm{D}^{+}\right) / N\left(\mathrm{~N}_{2} \mathrm{H}^{+}\right)$.

The obtained $\mathrm{H}_{2}$ column densities are given in Col. 4 of Table 7. The $\mathrm{N}_{2} \mathrm{H}^{+}$and $\mathrm{N}_{2} \mathrm{D}^{+}$column densities, fractional abundances, and the values of $R_{\text {deut }}$ are listed in Table 8 . The uncertainties in $N\left(\mathrm{~N}_{2} \mathrm{H}^{+}\right)$and $N\left(\mathrm{~N}_{2} \mathrm{D}^{+}\right)$were calculated by propagating the uncertainties in $T_{\text {ex }}, \tau_{\text {tot }}$, and $\Delta v$, and the uncertainties in $N\left(\mathrm{~N}_{2} \mathrm{D}^{+}\right) / N\left(\mathrm{~N}_{2} \mathrm{H}^{+}\right)$ratios are propagated from those in $N\left(\mathrm{~N}_{2} \mathrm{H}^{+}\right)$and $N\left(\mathrm{~N}_{2} \mathrm{D}^{+}\right)$.

\subsection{Ionization degree and cosmic-ray ionization rate}

The charge quasi-neutrality of plasma dictates that the number of positive and negative charges are equal. Since electrons are the dominant negative species, their fractional abundance nearly equals the sum of the abundances of positive ions, $x$ (cations $) \simeq x(\mathrm{e})$. Thus, one may obtain a lower limit to the ionization fraction by summing the abundances of several molecular ions:

$x(\mathrm{e})>x\left(\mathrm{~N}_{2} \mathrm{H}^{+}\right)+x\left(\mathrm{~N}_{2} \mathrm{D}^{+}\right)+x\left(\mathrm{H}_{3}^{+}\right)+x\left(\mathrm{H}_{2} \mathrm{D}^{+}\right)$.

In the following, we attempt to derive estimates of the cosmicray ionization rate and the fractional electron abundance using the abundances of $\mathrm{N}_{2} \mathrm{H}^{+}, \mathrm{N}_{2} \mathrm{D}^{+}$, and $\mathrm{H}_{2} \mathrm{D}^{+}$together with the reaction schemes and formulae presented in Crapsi et al. (2004) and Caselli et al. (2008). The rate coefficients for the $\mathrm{H}_{3}^{+}+\mathrm{H}_{2}$ isotopic system were calculated by Hugo et al. (2009). We used these for the deuteration sequence $\mathrm{H}_{3}^{+} \leftrightarrow$ $\mathrm{H}_{2} \mathrm{D}^{+} \leftrightarrow \mathrm{D}_{2} \mathrm{H}^{+} \leftrightarrow \mathrm{D}_{3}^{+}$(see their Table VIII). For other reactions, the rate coefficients have been adopted from the UMIST database ${ }^{8}$. The main difference between the Hugo et al. (2009) coefficients and those of Roberts et al. (2004) is that in the former, the effective backward rate coefficient, $k_{-1}$, of the reaction $\mathrm{H}_{3}^{+}+\mathrm{HD} \underset{k_{-1}}{\stackrel{k_{1}}{\rightleftharpoons}} \mathrm{H}_{2} \mathrm{D}^{+}+\mathrm{H}_{2}$, and the corresponding coefficients for multiply deuterated forms of $\mathrm{H}_{3}^{+}$are higher if the non-thermal ortho/para ratio of $\mathrm{H}_{2}$ (hereafter o/p- $\mathrm{H}_{2}$ ) is taken into account (Pagani et al. 1992; Gerlich et al. 2002; Flower et al. 2006a; Pagani et al. 2009a; Hugo et al. 2009).

The ortho- $\mathrm{H}_{2} \mathrm{D}^{+}$column density was derived towards Ori B9 $\mathrm{E}$ and $\mathrm{N}$ by Harju et al. (2006). Using their value, $N\left(\mathrm{o}-\mathrm{H}_{2} \mathrm{D}^{+}\right) \sim 3.0 \times 10^{12} \mathrm{~cm}^{-2}$, and the $\mathrm{H}_{2}$ column densities derived here, we measure $x\left(0-\mathrm{H}_{2} \mathrm{D}^{+}\right) \approx 8.0 \times 10^{-10}$ and $4.1 \times 10^{-10}$ towards Ori B9 $\mathrm{E}$ and $\mathrm{N}$, respectively ${ }^{9}$. The ortho/para ratio of $\mathrm{H}_{2} \mathrm{D}^{+}$(hereafter $\mathrm{o} / \mathrm{p}-\mathrm{H}_{2} \mathrm{D}^{+}$) depends heavily on $\mathrm{o} / \mathrm{p}-\mathrm{H}_{2}$. According to the model of Walmsley et al. (2004, see their Fig. 3), the characteristic steady-state value of o/p- $\mathrm{H}_{2}$ is $\sim 10^{-4}$ in the density range $n\left(\mathrm{H}_{2}\right) \sim 10^{5}-10^{6} \mathrm{~cm}^{-3}$ appropriate to objects in this study. This model deals with the situation of "complete depletion" and it is unclear how valid the quoted $\mathrm{o} / \mathrm{p}-\mathrm{H}_{2}$ is in less depleted gas. Pagani et al. (2009a) inferred high values of $\mathrm{o} / \mathrm{p}-\mathrm{H}_{2}\left(\sim 4 \times 10^{-3}-6 \times 10^{-2}\right)$ in L183.

For the moment, we adopt the value of o/p- $\mathrm{H}_{2}=10^{-4}$. The effect of increasing this ratio is examined briefly below. Assuming that $\mathrm{o} / \mathrm{p}-\mathrm{H}_{2} \mathrm{D}^{+}$is determined mainly by nuclear spin changing collisions with ortho- and para- $\mathrm{H}_{2}$, the quoted $\mathrm{o} / \mathrm{p}$ $\mathrm{H}_{2}$ ratio implies an $\mathrm{o} / \mathrm{p}-\mathrm{H}_{2} \mathrm{D}^{+}$of $\sim 0.7$ at $T=10 \mathrm{~K}$. The total

\footnotetext{
8 http://www.udfa.net/

9 Caselli et al. (2008) derived $N\left(\mathrm{o}-\mathrm{H}_{2} \mathrm{D}^{+}\right)=2.0 / 9.0 \times 10^{12} \mathrm{~cm}^{-2}$ toward position which is only $12^{\prime \prime} .7$ southeast of our line observations position Ori B9 $\mathrm{N}$, assuming a critical density $n_{\mathrm{cr}}=10^{5}$ and $10^{6} \mathrm{~cm}^{-3}$, respectively.
} 
Table 8. $\mathrm{N}_{2} \mathrm{H}^{+}$and $\mathrm{N}_{2} \mathrm{D}^{+}$column densities, fractional abundances, and the column density ratio.

\begin{tabular}{cccccc}
\hline \hline Position & $\begin{array}{c}N\left(\mathrm{~N}_{2} \mathrm{H}^{+}\right) \\
{\left[10^{12} \mathrm{~cm}^{-2}\right]}\end{array}$ & $\begin{array}{c}N\left(\mathrm{~N}_{2} \mathrm{D}^{+}\right) \\
{\left[10^{11} \mathrm{~cm}^{-2}\right]}\end{array}$ & $\begin{array}{c}x\left(\mathrm{~N}_{2} \mathrm{H}^{+}\right) \\
{\left[10^{-10}\right]}\end{array}$ & $\begin{array}{c}x\left(\mathrm{~N}_{2} \mathrm{D}^{+}\right) \\
{\left[10^{-11}\right]}\end{array}$ & $R_{\text {deut }} \equiv N\left(\mathrm{~N}_{2} \mathrm{D}^{+}\right) / N\left(\mathrm{~N}_{2} \mathrm{H}^{+}\right)$ \\
\hline IRAS 05405-0117 & $9.14 \pm 0.08^{a}$ & $3.19 \pm 0.37$ & 11.1 & 4.9 & $0.03 \pm 0.004$ \\
Ori B9 E & $4.54 \pm 0.65$ & $1.90 \pm 1.39$ & 6.9 & 5.0 & $0.04 \pm 0.03$ \\
Ori B9 N & $4.11 \pm 1.51$ & $1.46 \pm 0.65$ & 3.9 & 1.9 & $0.04 \pm 0.02$ \\
\hline
\end{tabular}

${ }^{a}$ Harju et al. (2006) estimated slightly lower $\mathrm{N}_{2} \mathrm{H}^{+}$column density, $\sim 6-8 \times 10^{12} \mathrm{~cm}^{-2}$, toward IRAS 05405-0117 from the $\mathrm{N}_{2} \mathrm{H}^{+}(1-0)$ data of Caselli \& Myers (1994).

${ }^{b}$ For the other velocity component $N\left(\mathrm{~N}_{2} \mathrm{H}^{+}\right)=1.56 \pm 0.09 \times 10^{12} \mathrm{~cm}^{-2}, N\left(\mathrm{~N}_{2} \mathrm{D}^{+}\right)=7.64 \pm 5.44 \times 10^{10} \mathrm{~cm}^{-2}$, and $R_{\text {deut }}=0.05 \pm 0.03$.

(ortho+para) $\mathrm{H}_{2} \mathrm{D}^{+}$abundances corresponding to this $\mathrm{o} / \mathrm{p}$ ratio are $x\left(\mathrm{H}_{2} \mathrm{D}^{+}\right) \approx 2.0 \times 10^{-9}$ and $1.0 \times 10^{-9}$ towards Ori B9 E and $\mathrm{N}$, respectively.

The $\mathrm{N}_{2} \mathrm{D}^{+} / \mathrm{N}_{2} \mathrm{H}^{+}$column density ratio, which we denote by $R_{\text {deut }}$, provides a rough estimate of the $\mathrm{H}_{2} \mathrm{D}^{+} / \mathrm{H}_{3}^{+}$abundance ratio, denoted here by $r$. According to the relation $R_{\text {deut }} \approx(r+$ $\left.2 r^{2}\right) /\left(3+2 r+r^{2}\right)$ derived by Crapsi et al. (2004; a more accurate formula is given in Eq. (13) of Caselli et al. 2008), the values of $R_{\text {deut }}$ given in Table 8 imply that $r \approx 0.08$ for IRAS $05405-0117$ and $r \approx 0.11$ for Ori B9 E and N. Using these $r$ values, we obtain the fractional abundances $x\left(\mathrm{H}_{3}^{+}\right)=x\left(\mathrm{H}_{2} \mathrm{D}^{+}\right) / r \approx 1.8 \times 10^{-8}$ (Ori B9 E) and $\approx 9.1 \times 10^{-9}$ (Ori B9 N). Substituting all the derived abundances into Eq. (7), we derive lower limits to the degree of ionization of $x(\mathrm{e})>2.0 \times 10^{-8}$ in Ori B9 E, and $>1.0 \times 10^{-8}$ in Ori B9 N.

Although the clump associated with IRAS 05405-0117 is not strongly evident in the ${ }^{13} \mathrm{CO}$ and $\mathrm{C}^{18} \mathrm{O}$ maps, a moderate $\mathrm{CO}$ depletion factor of 3.6 near Ori $\mathrm{B} 9 \mathrm{~N}$ has been derived (Caselli \& Myers 1995; Caselli et al. 2008). This estimate is based on ${ }^{13} \mathrm{CO}(1-0)$ observations completed with the FCRAO 14-m telescope (HPBW 50"), and a total $\mathrm{H}_{2}$ column density, $N\left(\mathrm{H}_{2}\right)$, derived from ammonia. By smoothing the LABOCA map to the resolution of $50^{\prime \prime}$, we obtain an average $\mathrm{H}_{2}$ column density of $1.3 \times 10^{22} \mathrm{~cm}^{-2}$ around Ori B9 N. This is a factor of 2.6 lower than the value adopted by Caselli et al. (2008). With this value of $N\left(\mathrm{H}_{2}\right)$, the fractional $\mathrm{CO}$ abundance, $x(\mathrm{CO})$, becomes $6.8 \times 10^{-5}$. The corresponding $\mathrm{CO}$ depletion factor, $f_{\mathrm{D}}$, is only 1.4 with respect to the often adopted fractional abundance from Frerking et al. (1982) ${ }^{10}$. The small value of $R_{\text {deut }}$ is consistent with a low CO depletion factor (e.g., Crapsi et al. 2004).

In chemical equilibrium, the fractional $\mathrm{H}_{3}^{+}$abundance is

$x\left(\mathrm{H}_{3}^{+}\right)=\frac{\zeta_{\mathrm{H}_{2}} / n\left(\mathrm{H}_{2}\right)+k_{-1} x\left(\mathrm{H}_{2} \mathrm{D}^{+}\right)}{D_{0}}$,

where $\zeta_{\mathrm{H}_{2}}$ is the cosmic-ray ionization rate of $\mathrm{H}_{2}$, and

$D_{0} \equiv k_{1} x(\mathrm{HD})+k_{\mathrm{CO}} x(\mathrm{CO})+k_{\mathrm{rec} 0} x(\mathrm{e})+k_{\mathrm{g}} x\left(\mathrm{~g}^{-}\right)+k_{\mathrm{N}_{2}} x\left(\mathrm{~N}_{2}\right)+\ldots$

The notation of Caselli et al. (2008) has been used here, i.e., $k_{1}$ and $k_{-1}$ are the forward and backward rate coefficients of the reaction mentioned above, and the other terms in $D_{0}$ refer to the destruction of $\mathrm{H}_{3}^{+}$in reactions with neutral molecules (e.g., $\mathrm{CO}$ and $\mathrm{N}_{2}$ ) and in recombination with electrons and on negatively charged dust grains.

By solving numerically Eqs. (8)-(10) and (13) of Caselli et al. (2008) together with our Eq. (8), we obtain the following estimates for the fractional electron abundance and cosmicray ionization rate: $x(\mathrm{e})=6.4 \times 10^{-7}, \zeta_{\mathrm{H}_{2}}=2.0 \times 10^{-16} \mathrm{~s}^{-1}$

\footnotetext{
10 The depletion factor is used in the text to express the fractional $\mathrm{CO}$ abundance with respect to the value $9.5 \times 10^{-5}$. Adopting a higher reference abundance (see Lacy et al. 1994) would not change the results of the calculations.
}

in Ori B9 E, and $x(\mathrm{e})=6.3 \times 10^{-7}, \zeta_{\mathrm{H}_{2}}=2.5 \times 10^{-16} \mathrm{~s}^{-1}$ in Ori B9 N. Here, we have used the $\mathrm{CO}$ depletion factor 1.4, and the dust parameters $\left(k_{\mathrm{g}^{-}}, x\left(\mathrm{~g}^{-}\right)\right.$quoted in Eqs. (11) and (12) of Caselli et al. (2008), which are based on a MRN dust-grain size distribution (Mathis et al. 1977) and effective grain recombination coefficients derived by Draine \& Sutin (1987). The average number densities, $\left\langle n\left(\mathrm{H}_{2}\right)\right\rangle$, derived in Sect. 4.2., were used to calculate the cosmic-ray ionization rates. The obtained values of $\zeta_{\mathrm{H}_{2}}$ are very similar to each other, as expected for such nearby cores (Williams et al. 1998; Bergin et al. 1999, and references therein).

For comparison, Bergin et al. (1999) found that adopting $\zeta_{\mathrm{H}_{2}}=5 \times 10^{-17} \mathrm{~s}^{-1}$ in their chemical model reproduced most closely their observations of the massive cores in Orion. We note that the "standard" value often quoted in the literature is $\zeta_{\mathrm{H}_{2}}=1.3 \times 10^{-17} \mathrm{~s}^{-1}$. The fractional electron abundances are also clearly higher than those calculated with the standard relation $x(\mathrm{e}) \sim 1.3 \times 10^{-5} n\left(\mathrm{H}_{2}\right)^{-1 / 2}$ (cf. McKee 1989; McKee et al. 1993), where the electron fraction is determined only by cosmic-ray ionization and $\zeta_{\mathrm{H}_{2}}$ has its above-mentioned standard value. The corresponding values would be $x(\mathrm{e}) \sim 5 \times$ $10^{-8}$ (Ori B9 E) and $x(\mathrm{e}) \sim 3 \times 10^{-8}$ (Ori B9 N). The mean value of the ionization degree found by Bergin et al. (1999) for the massive cores in Orion is $\sim 8 \times 10^{-8}$.

The parameters derived above depend on the adopted o/p- $\mathrm{H}_{2}$, which affects the backward-rate coefficient $k_{-1}, k_{-2}$, and $k_{-3}$ (see Caselli et al. 2008), and the CO depletion factor $f_{\mathrm{D}}$, which affects the destruction of $\mathrm{H}_{3}^{+}$. The fractional electron abundance can be decreased to $\sim 8 \times 10^{-8}$ by increasing o/p- $\mathrm{H}_{2}$ to $2.4 \times 10^{-3}$ (this yields a $\zeta_{\mathrm{H}_{2}}$ value of $1.3 \times 10^{-16} \mathrm{~s}^{-1}$ ). On the other hand, an increase in $f_{\mathrm{D}}$ will lead to a higher $x(\mathrm{e})$ but also lower $\zeta_{\mathrm{H}_{2}}$. A solution where both $x(\mathrm{e})$ and $\zeta_{\mathrm{H}_{2}}$ equal the respoective average values derived by Bergin et al. (1999) can be found by setting $f_{\mathrm{D}}$ to 4.4 and $\mathrm{o} / \mathrm{p}-\mathrm{H}_{2}$ to $3.4 \times 10^{-3}$.

However, the available observational data do not provide grounds for abandoning the present bona fide $f_{\mathrm{D}}$ value 1.4 , and the main uncertainty seems to be related to the unknown o/p- $\mathrm{H}_{2}$. Additional uncertainties in the $\zeta_{\mathrm{H}_{2}}$ values are caused by the approximate density estimates, and that densities in the positions observed in molecular lines are probably lower than the average densities adopted in the analysis. The electron abundance obtained assuming an $\mathrm{o} / \mathrm{p}-\mathrm{H}_{2}$ of $1.0 \times 10^{-4}$ is probably an upper limit. This o/p ratio corresponds to a steady state of highly depleted dense gas with high abundances of $\mathrm{H}^{+}$and $\mathrm{H}_{3}^{+}$capable of efficient proton exchange with $\mathrm{H}_{2}$ (Flower et al. 2007). Their replacement by other ions in less extreme situations can sustain higher $\mathrm{o} / \mathrm{p}-\mathrm{H}_{2}$ ratios.

A substantial amount of $\mathrm{CO}$ implies the presence of $\mathrm{HCO}^{+}$ in the gas. By including the dissociative electron recombination of $\mathrm{HCO}^{+}$, and the proton exchange reaction between $\mathrm{N}_{2} \mathrm{H}^{+}$ (or $\mathrm{N}_{2} \mathrm{D}^{+}$) and $\mathrm{CO}$ in the reaction scheme, the fractional $\mathrm{HCO}^{+}$ abundance can be solved. This estimate provides a slightly more 
Table 9. Parameters derived in Sect. 4.4.

\begin{tabular}{cccccccc}
\hline \hline Source & $x\left(\mathrm{H}_{2} \mathrm{D}^{+}\right)$ & $x\left(\mathrm{H}_{3}^{+}\right)$ & $x(\mathrm{e})_{l}{ }^{a}$ & $x(\mathrm{e})_{u}$ & $\langle x(\mathrm{e})\rangle^{b}$ & $\zeta_{\mathrm{H}_{2}}{ }^{c}$ & $C_{i}$ \\
& {$\left[10^{-9}\right]$} & {$\left[10^{-8}\right]$} & {$\left[10^{-8}\right]$} & {$\left[10^{-7}\right]$} & {$\left[10^{-7}\right]$} & {$\left[10^{-16} \mathrm{~s}^{-1}\right]$} & {$\left[10^{3} \mathrm{~cm}^{-3 / 2} \mathrm{~s}^{1 / 2}\right]$} \\
\hline Ori B9 E & 2.0 & 1.8 & $2.0 / 3.0$ & 6.4 & 3.4 & $2.0 / 1.0$ & 2.6 \\
Ori B9 N & 1.0 & 0.9 & $1.0 / 2.0$ & 6.3 & 3.3 & $2.5 / 1.3$ & 4.1 \\
\hline
\end{tabular}

${ }^{a}$ The first value is calculated from Eq. (7), whereas the second value is calculated from Eq. (9). ${ }^{b}$ This is the mean value between the lower and upper limit, where $x(\mathrm{e})_{l}$ is calculated from Eq. (9). ${ }^{c}$ The second value is derived by including $\mathrm{HCO}^{+}$in the analysis (see text).

stringent lower limit to the electron abundance than that imposed by Eq. (7) by requiring that

$$
\begin{aligned}
x(\mathrm{e}) \geq & x\left(\mathrm{H}_{3}^{+}\right)(1+r)+x\left(\mathrm{~N}_{2} \mathrm{H}^{+}\right)\left(1+R_{\text {deut }}\right) \\
& +x\left(\mathrm{HCO}^{+}\right)\left(1+R_{\text {deut }}\right) .
\end{aligned}
$$

Here it has been assumed that $\mathrm{N}_{2} \mathrm{H}^{+}$and $\mathrm{HCO}^{+}$have similar degrees of deuterium fractionation. By varying $\mathrm{o} / \mathrm{p}-\mathrm{H}_{2}$ until electron and the "known" cations are in balance, we obtain for $\mathrm{o} / \mathrm{p}-\mathrm{H}_{2}=2.7 \times 10^{-3}$, the lower limits $x(\mathrm{e}) \geq 3 \times 10^{-8}$ and $x(\mathrm{e}) \geq 2 \times 10^{-8}$ in Ori $\mathrm{B} 9 \mathrm{E}$ and $\mathrm{N}$, respectively. The corresponding values of $\zeta_{\mathrm{H}_{2}}$ are $1.0 \times 10^{-16} \mathrm{~s}^{-1}$ and $1.3 \times 10^{-16} \mathrm{~s}^{-1}$. In both solutions, $\mathrm{HCO}^{+}$is more abundant than $\mathrm{H}_{3}^{+}$, whereas for a high $x(\mathrm{e})\left(\right.$ low o/p- $\left.\mathrm{H}_{2}\right)$ the $\mathrm{HCO}^{+}$abundance is between those of $\mathrm{N}_{2} \mathrm{H}^{+}$and $\mathrm{H}_{2} \mathrm{D}^{+}$.

The obtained values of the cosmic-ray ionization rate vary smoothly with $\mathrm{o} / \mathrm{p}-\mathrm{H}_{2}$, and all viable solutions point towards $\zeta_{\mathrm{H}_{2}} \sim 1-2 \times 10^{-16} \mathrm{~s}^{-1}$. In the model of McKee (1989), these levels imply fractional ionizations of $\sim 1.1-1.6 \times 10^{-7}$ at the density $10^{5} \mathrm{~cm}^{-3}$. These values are between the lower and upper limits derived above. In what follows we assume that $x(\mathrm{e}) \sim 1 \times 10^{-7}$, keeping in mind that the true electron abundance is probably within a factor of a few of this value.

There is also uncertainty about the most abundant ion. According to our calculation, the electron abundance is an order of magnitude higher than the summed abundances of the positive ions $\mathrm{H}_{3}^{+}, \mathrm{HCO}^{+}$, and $\mathrm{N}_{2} \mathrm{H}^{+}$for $\mathrm{o} / \mathrm{p}-\mathrm{H}_{2}=1.0 \times 10^{-4}$, whereas for the higher $\mathrm{o} / \mathrm{p}-\mathrm{H}_{2}$ ratio $x\left(\mathrm{HCO}^{+}\right)$is comparable to $x(\mathrm{e})$. This suggests that in the first case, the reaction scheme misses the most abundant cation(s). In depleted regions with densities below $10^{6} \mathrm{~cm}^{-3}$, protons, $\mathrm{H}^{+}$, are likely to be the dominant ions (Walmsley et al. 2004; Pagani et al. 2009a). On the other hand, as discussed in Crapsi et al. (2004) and references therein, if atomic oxygen is abundant in the gas phase the major ion may be $\mathrm{H}_{3} \mathrm{O}^{+}$. To our knowledge, this ion has not yet been found in cold clouds (see also Caselli et al. 2008). When discussing the ambipolar diffusion timescale in Sect. 5.6, we assume that the most abundant ion is either $\mathrm{H}^{+}$or $\mathrm{HCO}^{+}$.

Furthermore, we estimate the value of a constant, $C_{i}$, that describes the relative contributions of molecular ions and metal ions to the ionization balance (Williams et al. 1998, their Eq. (4); Bergin et al. 1999; Padoan et al. 2004). The value of $C_{i}$ can be used to estimate the strength of the ion-neutral coupling in terms of the wave-coupling parameter, $W \propto C_{i}$ (see Sect. 5.7). In this analysis, it is assumed that the electron abundance is determined by cosmic-ray ionization balanced by recombination and is appropriate for cores where $A_{\mathrm{V}}>4 \mathrm{mag}$ (i.e., ionization due to cosmic rays dominates over that resulting from UV radiation). Adopting the electron abundance $1 \times 10^{-7}$ and $\zeta_{\mathrm{H}_{2}} \sim 10^{-16} \mathrm{~s}^{-1}$, we find values of $C_{i} \simeq 2.6-4.1 \times 10^{3} \mathrm{~cm}^{-3 / 2} \mathrm{~s}^{1 / 2}$ in our cores. These are similar to the value found by Bergin et al. (1999) for the massive cores in Orion $\left(3.6 \times 10^{3} \mathrm{~cm}^{-3 / 2} \mathrm{~s}^{1 / 2}\right)$. McKee (1989) derives $C_{i}=3.2 \times 10^{3} \mathrm{~cm}^{-3 / 2} \mathrm{~s}^{1 / 2}$ for an idealised model of cosmic-ray ionization and Williams et al. (1998) obtained
$C_{i}=2.0 \times 10^{3} \mathrm{~cm}^{-3 / 2} \mathrm{~s}^{1 / 2}$ for low-mass cores. All the parameters derived in this section are summarised in Table 9.

\section{Discussion}

In the following, we discuss the results presented in the previous sections, and compare them with the results from the literature.

\subsection{Nature of submm sources in Ori B9}

By combining the submm LABOCA and far-infrared Spitzer data, we can distinguish between starless cores and protostellar cores. In addition to the four IRAS sources in the region, two of the new submm sources, namely SMM 3 and SMM 4, are clearly associated with Spitzer point sources and are protostellar. The remaining six submm cores are starless.

IRAS 05399-0121 was previously classified as a Class I protostar (Bally et al. 2002, and references therein). However, taking into account the rather low bolometric $(18.5 \mathrm{~K})$ and kinetic temperatures (13.7 K, Harju et al. 1993), and high values of $L_{\text {submm }} / L_{\text {bol }}(2 \%)$ and $M_{\text {tot }} / L_{\text {bol }}^{0.6}\left(0.45 M_{\odot} / L_{\odot}^{0.6}\right)$, we suggest the source is in a transition phase from Class 0 to Class I (see Bontemps et al. 1996; Froebrich 2005). This source is associated with the highly collimated jet HH 92 (Bally et al. 2002).

The SED of IRAS 05413-0104 derived here is consistent with its previous classification as a Class 0 object (e.g., Cabrit et al. 2007, and references therein). The source is associated with the highly symmetric jet HH 212 (Lee et al. 2006, 2007; Codella et al. 2007; Smith et al. 2007; Cabrit et al. 2007). IRAS 05412-0105 and IRAS 05405-0117 have very similar SEDs, and they, too, are likely to represent the Class 0 stage. The weak-line wings in the $\mathrm{N}_{2} \mathrm{H}^{+}(1-0)$ hyperfine lines of IRAS 05405-0117 (see Fig. 4, top) could indicate the presence of outflow from an embedded protostellar object.

The $L_{\text {submm }} / L_{\text {bol }}$ ratio for both SMM 3 and SMM 4 is $11 \%$, which with the low values of $T_{\text {bol }}$ imply that these new submm sources are Class 0 candidates (e.g., Froebrich 2005, and references therein) deeply embedded in a massive, cold envelope. On a bolometric luminosity versus temperature diagram these objects lie on the evolutionary track for a Class 0 source with initially massive envelope (see Fig. 12 in Myers et al. 1998).

The starless cores SMM 1, 2, 5, 6, 7, and Ori B9 N, are likely to be prestellar because their densities are relatively high (0.6-5.8 $\times 10^{5} \mathrm{~cm}^{-3}$; see also Sect. 4.2). The $24 \mu \mathrm{m}$ Spitzer source close to SMM 5 is probably not associated with this core, but is further from the core centre and not detected at $70 \mu \mathrm{m}$ (Fig. 2).

There are equal numbers of prestellar and protostellar cores in Ori B9. This situation is similar to that found by Enoch et al. (2008) in Perseus, Serpens, and Ophiuchus, and suggests that the lifetimes of prestellar and protostellar cores are comparable. Evolutionary timescales are further discussed in Sect. 5.6. 


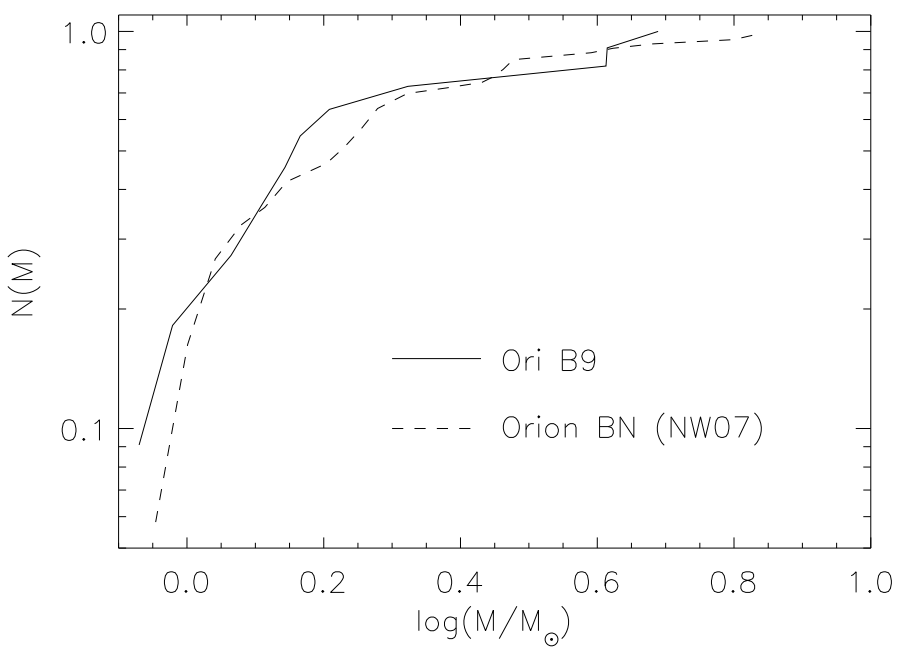

Fig. 7. Normalised cumulative mass functions, $\mathcal{N}(M)$, for the prestellar cores in Ori B9 (solid line) and in Orion B North (dashed line) studied by Nutter \& Ward-Thompson (2007).

\subsection{Mass distribution and core separations}

The spatial and mass distribution of cores are both important parameters concerning the cloud fragmentation mechanism. Our core sample is, however, so small that it is not reasonable to study the properties of these distributions directly. Therefore, we only compared them with the distributions derived for another, larger core sample in Orion GMC by NW07. We compare particularly with data for Orion B North because the SCUBA $850 \mu \mathrm{m}$ map of Orion B North (see Fig. 2c in NW07) looks qualitatively similar to Ori B9. The data of a Orion B North also has deeper sensitivity and completeness limit than other regions studied by NW07, and the region also contains a large number of cores. Figure 7 presents the observed cumulative mass functions, which include cores of mass less than $M$, i.e., $\mathcal{N}(M)=N(m<M) / N_{\text {tot }}$, for both the core masses in Ori B9 and masses of prestellar cores in Orion B North derived by NW07. We note that the core mass function (CMF) studied by NW07 is constructed by removing the Class I protostars from the sample, so that CMF includes only cores that initially have all their mass available for star formation. Correspondingly, we excluded IRAS 05399-0121 from our sample. We also multiplied the core masses by the required factors when comparing with NW07 values, because of differences in the assumed values of $T_{\text {dust }}, \kappa_{\lambda}$, and distance (NW07 used the following values: $T_{\text {dust }}=20 \mathrm{~K}, \kappa_{850}=0.1 \mathrm{~m}^{2} \mathrm{~kg}^{-1}$, and $d=400 \mathrm{pc}$ ).

To determine whether the two datasets are derived from the same core mass distribution, we carried out the KolmogorovSmirnov (K-S) test. The K-S test yields the maximum vertical difference between the cumulative distributions of $D=0.166$, and a probability of approximately $95 \%$ that the core mass distributions in Ori B9 and Orion B North are drawn from the same parent distribution.

Figure 8 (top) shows the observed core-separation distribution and the distribution expected for the same number of randomly positioned cores over an identical area $\left(0.22 \mathrm{deg}^{2}\right)$. The mean and median of the core separations in Ori B9 are $\log (r / \mathrm{AU})=5.47 \pm 0.09\left(2.9 \pm 0.6 \times 10^{5} \mathrm{AU}\right)$ and $5.42(2.6 \times$ $\left.10^{5} \mathrm{AU}\right)$, respectively. The quoted error in the mean corresponds to the standard deviation. These values are similar to those of randomly positioned cores, for which the mean and median are $\log (r / \mathrm{AU})=5.59 \pm 0.05$ and $5.59 \pm 0.06$,
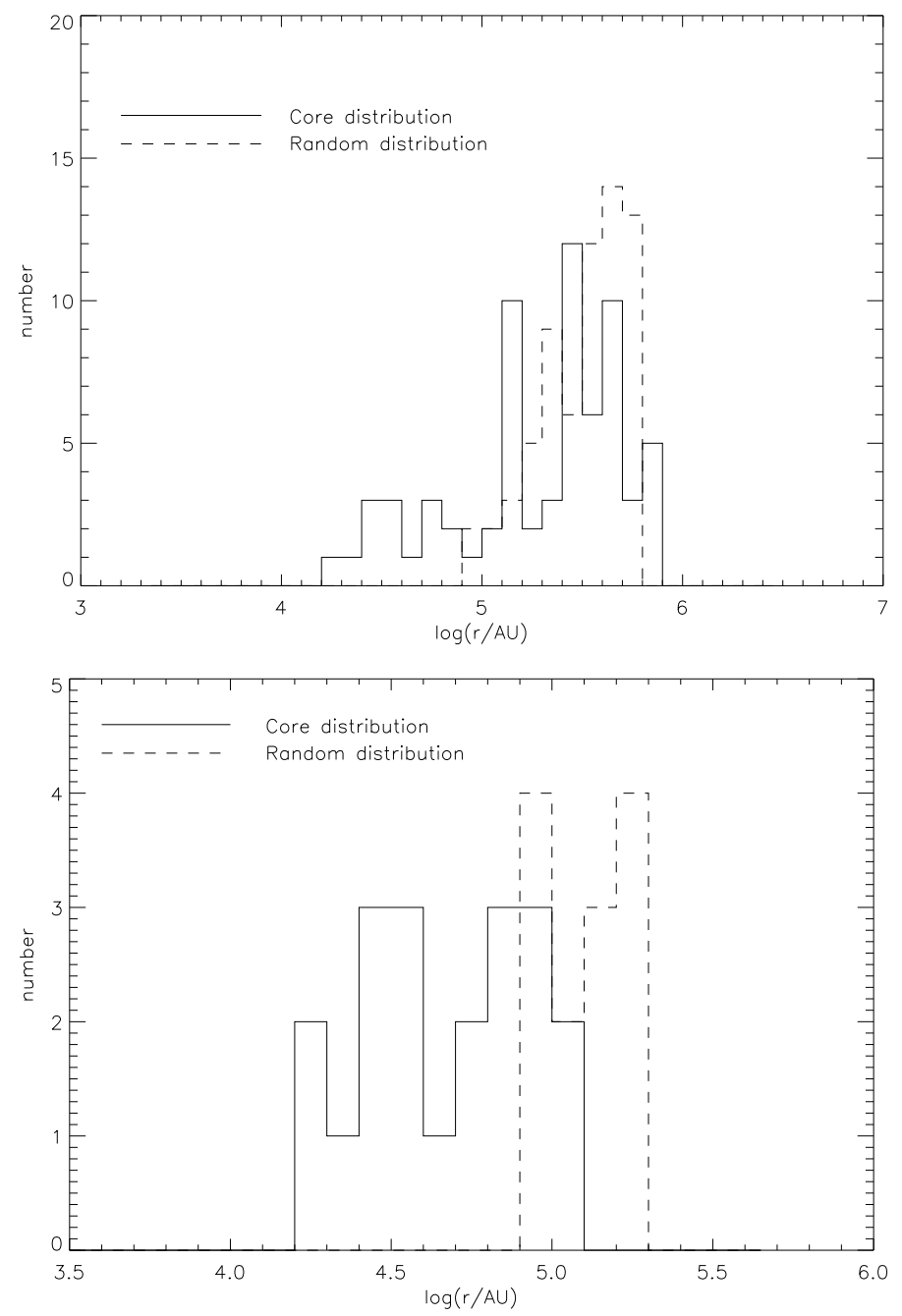

Fig. 8. Top: observed core separation distribution (solid line) compared with the expected distribution for random distribution of the same number of sources as the observed sample over an identical area (dashed line). Bottom: observed nearest-neighbour distribution (solid line) compared with the expected distribution for random distribution of the same number of sources as the observed sample over the same area (dashed line).

respectively. The quoted uncertainties are the standard deviation of the sampling functions. For the core-separation distribution in Orion B North studied by NW07, the corresponding values are $5.67 \pm 0.03$ and 5.63 , suggesting that the fragmentation scale is similar in both Ori B9 and Orion B North. Similar fragmentation scales and because the CMFs resemble the stellar IMF (Goodwin et al. 2008) suggest that the origin of cores in these two regions is probably determined by turbulent fragmentation (e.g., Mac Low \& Klessen 2004; Ballesteros-Paredes et al. 2007). The clustered mode of star formation in these two regions suggests that turbulence is driven on large scales (e.g., Klessen 2001). Enoch et al. (2007) found the median separations of $\log (r / \mathrm{AU})=3.79,4.41$, and 4.36 in nearby molecular clouds Ophiuchus, Perseus, and Serpens, respectively. The spatial resolution of the Bolocam (31") used by Enoch et al. (2007) at the distance of Ophiuchus, Perseus, and Serpens, is $0.02,0.04$, and $0.04 \mathrm{pc}$. The latter two are similar to our resolution. These results suggest that the fragmentation scales in Perseus and Serpens differ from those in Orion. 
Figure 8 (bottom) compares the observed nearest-neighbour distribution with the distribution for randomly positioned cores. The mean and median of the nearest-neighbour distribution in Ori B9 are $\log (r / \mathrm{AU})=4.75 \pm 0.09\left(5.6 \pm 1.3 \times 10^{4} \mathrm{AU}\right)$ and $4.62\left(4.2 \times 10^{4} \mathrm{AU}\right)$, respectively. These values are rather different from those expected for random distributions, for which the mean and median are $\log (r / \mathrm{AU})=5.08 \pm 0.08$ and $5.07 \pm 0.11$, respectively. For core positions in Orion B North, the mean and median are $\log (r / \mathrm{AU})=4.46 \pm 0.03$ and 4.35 , respectively (NW07). This comparison also supports the idea that the scale of fragmentation, and the amount of clustering are similar in Ori B9 and Orion B North. We note that the minimum observable separation corresponds to the beam size, i.e., $18^{\prime \prime}$. 6 or $\sim 8.3 \times 10^{3} \mathrm{AU}$ at $450 \mathrm{pc}$. We also note that the source sample is too small to measure the significance of the clustering in Ori B9 based on the two-point correlation function.

\subsection{Sizes, shapes, and density structures of the cores}

Starless cores in Ori B9, for which the mean value of the deconvolved angular size in units of the beam $F W H M$ is $\left\langle\theta_{\mathrm{s}} / \theta_{\text {beam }}\right\rangle=2.5 \pm 0.8$, are larger on average than protostellar cores $\left(\left\langle\theta_{\mathrm{s}} / \theta_{\text {beam }}\right\rangle=1.6 \pm 0.2\right)$. These sizes are similar to those found by Enoch et al. (2008) in Perseus $\left(\left\langle\theta_{\mathrm{s}} / \theta_{\text {beam }}\right\rangle=2.2\right.$ and 1.6 for starless and protostellar cores, respectively). The mean axis ratios at half-maximum contours of starless and protostellar cores are also 2.5 and 1.6, respectively (see Table 2, Col. (7)). This indicates that starless cores in Ori B9 are also more elongated on average than protostellar cores (cf. Offner \& Krumholz 2009).

These values of $\theta_{\mathrm{s}} / \theta_{\text {beam }}$ can be used to infer the steepness of the core radial density profile (Young et al. 2003; Enoch et al. 2008). According to the correlation between $\theta_{\mathrm{s}} / \theta_{\text {beam }}$ and density-power-law index, $p$, found by Young et al. (2003, see their Fig. 27) mean $\theta_{\mathrm{s}} / \theta_{\text {beam }}$ values of 2.5 for starless and 1.6 for protostellar cores imply an average index of $p \sim 0.9-1.0$ and 1.4-1.5, respectively. Moreover, Fig. 25 of Young et al. (2003) suggest power-law indices $<1$ for starless cores and 1.1-1.6 for protostellar cores, consistent with those inferred by the average deconvolved angular source sizes. The low values of $p \sim 1.0$ for starless cores suggest that they are modelled most accurately by shallower density profiles than the protostellar cores. These results agree with those found by Ward-Thompson et al. (1999) using $1.3 \mathrm{~mm}$ dust continuum data, and Caselli et al. (2002b) using $\mathrm{N}_{2} \mathrm{H}^{+}(1-0)$ maps.

\subsection{Deuterium fractionation and depletion in the IRAS $05405-0117$ region}

The $\mathrm{N}_{2} \mathrm{D}^{+} / \mathrm{N}_{2} \mathrm{H}^{+}$column-density ratio, $R_{\text {deut }}$, is supposed to increase strongly as the core evolves (Caselli 2002; Crapsi et al. 2005a, their Fig. 5; Fontani et al. 2006; Emprechtinger et al. 2009; but see Roberts \& Millar 2007). This can be understood as the abundances of $\mathrm{H}_{3}^{+}$, and its deuterated forms, which transfer deuterium to other molecules, increase with increasing density because of molecular depletion and a lower degree of ionization. Crapsi et al. (2005a) suggested that prestellar cores are characterised by $R_{\text {deut }}>0.1$, whereas starless cores with $R_{\text {deut }}<0.1$ are not necessarily sufficiently dense for CO to be heavily depleted (Roberts \& Millar 2007). It should be noted that in cores without internal heating sources the degree of deuterium fractionation is likely to increase inwards as the density increases and temperature decreases due to the attenuation of starlight. This temporal and radial tendency is likely to be reversed during the core collapse because of compressional heating and the formation of a protostar (e.g., Aikawa et al. 2008a; see also Fig. 3 in Emprechtinger et al. 2009).

The positions studied here have $R_{\text {deut }} \sim 0.03-0.04$. This is $\sim 2-3 \times 10^{3}$ times higher than the cosmic D/H elemental abundance of $\sim 1.5 \times 10^{-5}$ (Linsky et al. 1995; 2006; Oliveira et al. 2003). The $\mathrm{H}_{2} \mathrm{D}^{+} / \mathrm{H}_{3}^{+}$ratios that we derived are also $\sim 7 \times 10^{3}$ times higher than the cosmic $\mathrm{D} / \mathrm{H}$ ratio. Our $R_{\text {deut }}$ values are similar to those found by Crapsi et al. (2005a) toward several low-mass starless cores, and to those found by Emprechtinger et al. (2009) toward Class 0 sources. Like Emprechtinger et al. (2009), we find that the deuterium fractionation of $\mathrm{N}_{2} \mathrm{H}^{+}$in protostellar cores, which takes place in the cold extended envelope, is similar to that in prestellar cores. It has been found that the values of $R_{\text {deut }}$ toward high-mass star-forming cores are (usually) lower than those found in the present study (Fontani et al. 2006; see also Emprechtinger et al. 2009). This is consistent with our sources being low- to intermediate-mass star-forming cores.

As discussed by Walmsley et al. (2004) and Flower et al. (2006a), the $\mathrm{H}_{2} \mathrm{D}^{+}$abundance depends (inversely) on the ortho:para ratio of $\mathrm{H}_{2}$, because the reaction $\mathrm{H}_{2} \mathrm{D}^{+}+\mathrm{H}_{2} \stackrel{k_{-1}}{\rightarrow} \mathrm{H}_{3}^{+}+\mathrm{HD}$ is rapid between ortho forms. The ortho:para ratio of $\mathrm{H}_{2}$ decreases with time and gas density, and is therefore high at early stages of core evolution. Consequently, a relatively high degree of deuterium fractionation is a sign of matured chemistry characterised by a low ortho:para ratio of $\mathrm{H}_{2}$ and probably a high degree of molecular depletion. A low $\mathrm{CO}$ depletion factor of 1.4 close to $\mathrm{N}_{2} \mathrm{H}^{+}$peak Ori B9 $\mathrm{N}$ (see Sect. 4.4) is consistent with the $R_{\text {deut }}$ value of Ori B9 N (e.g., Crapsi et al. 2004; see also Fig. 4 in Emprechtinger et al. 2009). The ortho- $\mathrm{H}_{2} \mathrm{D}^{+}$detection towards Ori B9 E and N suggests an evolved chemical stage and is indicative of a long-lasting prestellar phase. The non-detection toward IRAS 05405-0117 can be explained by a lower ortho$\mathrm{H}_{2} \mathrm{D}^{+}$abundance due to the central heating by the protostar.

\subsection{Evidence of a $\mathrm{N}_{2} \mathrm{H}^{+}$"hole" and chemical differentiation}

The $\mathrm{N}_{2} \mathrm{H}^{+}$map of Caselli \& Myers (1994, see their Fig. 2) and sub-mm dust continuum map of the clump associated with IRAS 05405-0117 (Fig. 3) are not extremely similar. The strongest dust continuum peak, SMM 4, does not stand out in $\mathrm{N}_{2} \mathrm{H}^{+}$. Moreover, the northern $\mathrm{N}_{2} \mathrm{H}^{+}$maximum, Ori B9 $\mathrm{N}$, seem to be shifted with respect to the northern dust peak, and the $\mathrm{N}_{2} \mathrm{H}^{+}$ peak Ori B9 E does not correspond to any dust emission peak.

To determine whether the $\sim 1.5$ times higher resolution of the LABOCA $870 \mu \mathrm{m}$ map relative to the $\mathrm{N}_{2} \mathrm{H}^{+}$map of Caselli \& Myers (1994) explains the different appearance of the dust continuum and $\mathrm{N}_{2} \mathrm{H}^{+}$maxima, we smoothed the LABOCA map to a resolution similar to that of the $\mathrm{N}_{2} \mathrm{H}^{+}$map (27"). The smoothed $870 \mu \mathrm{m}$ map, however, still shows the same differences between the dust continuum and $\mathrm{N}_{2} \mathrm{H}^{+}$.

The Class 0 candidate SMM 4 (see Sects. 4.1 and 5.1) can represent an extreme case of depletion, where $\mathrm{N}_{2} \mathrm{H}^{+}$has also disappeared from the gas phase due to freeze-out on to the dust grain surfaces. There is evidence of $\mathrm{N}_{2} \mathrm{H}^{+}$depletion in the centres of chemically evolved cores, such as B68 (Bergin et al. 2002), L1544 (Caselli et al. 2002a), L1512 (Lee et al. 2003), and L1521F (Crapsi et al. 2004). Example of the $\mathrm{N}_{2} \mathrm{H}^{+}$depletion toward Class 0 source is IRAM 04191+1522 in Taurus (Belloche \& André 2004). Pagani et al. (2005) found clear signs of moderate $\mathrm{N}_{2} \mathrm{H}^{+}$depletion in the prestellar core L183 (see also Pagani et al. 2007). Schnee et al. (2007) also 
found clear evidence of $\mathrm{N}_{2} \mathrm{H}^{+}$depletion toward the dust centre of TMC-1C. We note that SMM 4 is probably not warm enough for $\mathrm{CO}$ to evaporate from the grain mantles $(\sim 20 \mathrm{~K}$, e.g., Aikawa et al. 2008a), so it is unlikely that $\mathrm{CO}$, which is the main destroyer of $\mathrm{N}_{2} \mathrm{H}^{+}$(through reaction $\mathrm{N}_{2} \mathrm{H}^{+}+\mathrm{CO} \rightarrow \mathrm{HCO}^{+}+\mathrm{N}_{2}$ ), caused the disappearance of $\mathrm{N}_{2} \mathrm{H}^{+}$from the gas phase.

To study the chemical variations within the clump, we compared our previously determined $\mathrm{NH}_{3}$ column densities with the present $\mathrm{N}_{2} \mathrm{H}^{+}$column densities. The integrated $\mathrm{NH}_{3}(1,1)$ and $(2,2)$ intensity maps of the clump (see Appendix A in Harju et al. 1993) show roughly the same morphology as the submm map. The $\mathrm{NH}_{3}$ column densities toward IRAS 05405-0117, Ori B9 E, and Ori B9 $\mathrm{N}$ are $11.9 \pm 1.3 \times 10^{14}, 7.2 \pm 1.3 \times 10^{14}$, and $9.7 \pm 4.6 \times$ $10^{14} \mathrm{~cm}^{-2}$, respectively. The corresponding $\mathrm{NH}_{3} / \mathrm{N}_{2} \mathrm{H}^{+}$column density ratios are about $130 \pm 14,159 \pm 37$, and $236 \pm 87$. These values suggest that the $\mathrm{NH}_{3} / \mathrm{N}_{2} \mathrm{H}^{+}$abundance ratio is higher towards starless condensations than towards the IRAS source. Hotzel et al. (2004) found a similar tendency in B217 and L1262: the $\mathrm{NH}_{3} / \mathrm{N}_{2} \mathrm{H}^{+}$abundance ratios are at least twice as large in the dense starless parts of the cores than in the regions closer to the YSO (see Caselli et al. 2002b, for other low-mass star-forming regions). The same trend is also found in the high-mass starforming region IRAS 20293+3952 (Palau et al. 2007). This is in accordance with chemistry models (Aikawa et al. 2005) and previous observations (Tafalla et al. 2004), which suggest that $\mathrm{NH}_{3}$ develops slightly later than $\mathrm{N}_{2} \mathrm{H}^{+}$, and can resist depletion up to higher densities. It should be noted that models by Aikawa et al. (2005) reproduce the observed enhancement of the $\mathrm{NH}_{3} / \mathrm{N}_{2} \mathrm{H}^{+}$ratio by adopting the branching ratio for the dissociative recombination of $\mathrm{N}_{2} \mathrm{H}^{+}$measured by Geppert et al. (2004; i.e., $\mathrm{N}_{2} \mathrm{H}^{+}+\mathrm{e} \rightarrow \mathrm{NH}+\mathrm{N}$ accounts for $64 \%$ of the total reaction). However, this branching ratio has been retracted by the same authors ${ }^{11}$, and thus the cause of the increase in the $\mathrm{NH}_{3} / \mathrm{N}_{2} \mathrm{H}^{+}$ abundance ratio is unclear at the moment.

\subsection{Core evolution: quasi-static versus dynamic}

The degree of ionization in dense cores determines the importance of magnetic fields in the core dynamics. The ionization fractions in low-mass cores are found to be $10^{-8}<x(\mathrm{e})<10^{-6}$ (Caselli et al. 1998; Williams et al. 1998). The physical origin of the large variations in $x(\mathrm{e})$ is not well understood, although variations in $\zeta_{\mathrm{H}_{2}}$ or appropriate values of metal depletion are assumed (Padoan et al. 2004). Padoan et al. (2004) suggested that the observed variations in $x(\mathrm{e})$ can be understood as the combined effect of variations in core age, extinction, and density.

Fractional ionizations can be transformed into estimates of the ambipolar diffusion (AD) timescale, $\tau_{\mathrm{AD}}$. For this purpose, we used Eq. (5) of Walmsley et al. (2004). Assuming that $\mathrm{H}^{+}$is the dominant ion (see Sect. 4.4), one obtains $\tau_{\mathrm{AD}} \sim$ $8 \times 10^{13} x(\mathrm{e}) \mathrm{yr}$. If the dominant ion is $\mathrm{HCO}^{+}, \tau_{\mathrm{AD}} \sim 1.3 \times$ $10^{14} x(\mathrm{e}) \mathrm{yr}$, i.e., $\sim 60 \%$ longer than in the former case. Using the electron abundance $x(\mathrm{e})=1 \times 10^{-7}$, we obtain $\tau_{\mathrm{AD}} \sim$ $10^{7} \mathrm{yr}$. This timescale is roughly 70 and 100 times longer than the free-fall time $\left(\tau_{\mathrm{ff}} \sim 3.7 \times 10^{7}\left(n\left(\mathrm{H}_{2}\right)\left[\mathrm{cm}^{-3}\right]\right)^{-1 / 2} \mathrm{yr}\right)$ of Ori B9 E and N, respectively. Since $\tau_{\mathrm{AD}}>\tau_{\mathrm{ff}}$, the cores may be supported against gravitational collapse by magnetic fields and ion-neutral coupling. The magnetic field that is needed to support the cores can be estimated using the relation between the critical mass required for collapse and the magnetic

\footnotetext{
11 Their laboratory experiment suggests that the above-mentioned branching ratio is only $10 \%$ (see Aikawa et al. 2008b).
}

flux (see Eq. (2) in Mouschovias \& Spitzer 1976). Using the masses and radii from Table 7 , we obtain a critical magneticfield strength of $\sim 80 \mu \mathrm{G}$ for Ori B9 E/IRAS 05405-0117 and $\sim 200 \mu \mathrm{G}$ for Ori B9 N. These are rather high values compared to those that have been observed (Troland et al. 1996; Crutcher 1999; Crutcher \& Troland 2000; Crutcher et al. 2004; Turner \& Heiles 2006; Troland \& Crutcher 2008).

According to the "standard" model of low-mass star formation, $\tau_{\mathrm{AD}} / \tau_{\mathrm{ff}} \sim 10$ (see, e.g., Shu et al. 1987; Ciolek \& Basu 2001, and references therein). Since AD is generally a slow process, the core evolution toward star formation occur quasistaticly. The chemical abundances found in the present study $\left(x\left(\mathrm{~N}_{2} \mathrm{H}^{+}\right) \sim 10^{-10}, x\left(\mathrm{NH}_{3}\right) \sim 10^{-7}\right.$, see Sect. 5.8) are consistent with chemical models for a dynamically young, but chemically evolved (age > $10^{5}$ yr) source (Bergin \& Langer 1997; Roberts et al. 2004; Aikawa et al. 2005; Shirley et al. 2005; see also Kirk et al. 2007, and references therein). This supports the idea that the sources have been static or slowly contracting for more than $10^{5} \mathrm{yr}$, and conforms with the estimated AD timescales.

On the other hand, the equal numbers of prestellar and protostellar cores suggest that the prestellar core lifetime should be similar to the lifetime of embedded protostars. Since the duration of the protostellar stage is $\sim$ few $\times$ $10^{5}$ yr (e.g., Ward-Thompson et al. 2007; Hatchell et al. 2007; Galván-Madrid et al. 2007; Enoch et al. 2008), the prestellar core evolution should be rather dynamic and last for only a few free-fall times, as is the case in star formation driven by supersonic turbulence (e.g., Mac Low \& Klessen 2004; Ballesteros-Paredes et al. 2007). This seems to contradict with the above results of $\mathrm{AD}$ timescales. However, to recognise the cores in the sub-mm map, they are presumed to be in the highdensity stage of their evolution. Thus, the short statistical lifetime deduced above is still consistent with the quasi-static evolution driven by $\mathrm{AD}$, if we only observe the densest stages of a longer timescale core evolution (e.g., Enoch et al. 2008; Crutcher et al. 2009). Also, the dynamic phase of the core evolution for which $\tau_{\mathrm{AD}}$ equals only a few free-fall times might be appropriate for magnetically near-critical (the mass-to-magnetic flux ratio being $\sim 80 \%$ of the critical value) or already slightly supercritical cores when rapid collapse ensues (Ciolek \& Basu 2001; see also Tassis \& Mouschovias 2004).

\subsection{Line-widths and turbulence}

The $\mathrm{N}_{2} \mathrm{D}^{+}$line-widths in Ori B9 $\mathrm{E}$ and $\mathrm{N}$ are significantly narrower than the $\mathrm{N}_{2} \mathrm{H}^{+}$line-widths (by factors of $\sim 1.5$ and $\sim 1.9$, respectively). Crapsi et al. (2005a) found similar trends in several low-mass starless cores (see their Table 4). This is probably because $\mathrm{N}_{2} \mathrm{D}^{+}$traces the high density nuclei of starless cores, where non-thermal turbulent motions are expected to be insignificant (e.g., André et al. 2007; Ward-Thompson et al. 2007).

The non-thermal component dominate the $\mathrm{N}_{2} \mathrm{H}^{+}$line-widths in the observed positions (the thermal line-width of $\mathrm{N}_{2} \mathrm{H}^{+}$is about $0.126 \mathrm{~km} \mathrm{~s}^{-1}$ at $10 \mathrm{~K}$, and thus $\Delta v_{\mathrm{NT}} / \Delta v_{\mathrm{T}} \sim 2$ ). However, the level of internal turbulence, as estimated from the ratio of the non-thermal velocity dispersion to the isothermal speed of sound (e.g., Kirk et al. 2007), is not dynamically significant.

Using Eq. (7) of Williams et al (1998) and the derived values for the molecular/metal ion-contribution constant $C_{i}$ and cosmicray ionization rate $\zeta_{\mathrm{H}_{2}}$ (see Table 9), we see that non-thermal $\mathrm{N}_{2} \mathrm{H}^{+}$line broadening in the observed positions can be explained in part by magnetohydrodynamic (MHD) wave propagation. The 
large wave-coupling parameters in our sources $(W \gg 1)$ suggest that the coupling between the field and gas is strong and the waves are not suppressed. The derived values of $W(\sim 30-60$, in the case of minimum turbulence) agree with the $\tau_{\mathrm{AD}} / \tau_{\mathrm{ff}}$ ratios (Williams et al. 1998). The estimated degrees of coupling between the magnetic field and gas also conforms with the susceptibility to fragmentation (Bergin et al. 1999).

Caselli \& Myers (1995) analysed ammonia cores in the Orion B GMC and found an inverse relationship between core line-width and distance to the nearest stellar cluster. The nearest stellar cluster to Ori B9 is NGC 2024 at the projected distance of 5.2 pc (see Sect. 1.1.), so its role in driving external turbulence in the region is probably insignificant.

\subsection{Formation of a small stellar group in Ori B9}

Internal turbulence or gravitational motions in massive molecular-cloud cores may promote fragmentation of the medium. This can easily generate sheets and filaments (e.g., Caselli \& Myers 1995; André et al. 2008). The collapse of these elongated clumps most probably results in the formation of a small stellar group or a binary system rather than a single star (e.g., Launhardt et al. 1996). Only the densest parts of the filaments, the dense cores, are directly involved in star formation. It is unclear at the present time whether the collapse of an individual prestellar core typically produces single stars or multiple protostellar systems (see André et al. 2008).

The total mass of gas and dust of the clump associated with IRAS 05405-0117 as derived from the dust continuum emission is $\sim 14 M_{\odot}$, and it has an elongated structure with multiple cores (local maxima in the filament are separated by more than one beam size, see Fig. 3). The previous mass estimates by Harju et al. (1993) based on $\mathrm{NH}_{3}$ were much higher: $\sim 50 M_{\odot}$ derived from $N\left(\mathrm{NH}_{3}\right)$ distribution, and $\sim 310 M_{\odot}$ derived from peak local density. The uncertainty in the abundance ${ }^{12}$ and lower resolution used are certainly affecting the estimation of the mass from $\mathrm{NH}_{3}$. However, the clump has enough mass to form a small stellar group.

The kinetic temperature, velocity dispersion, and the fractional $\mathrm{H}_{2} \mathrm{D}^{+}$abundance in the clump are similar to those in the well-studied prestellar cores, e.g., L1544 and L183, where strong emission of $\mathrm{H}_{2} \mathrm{D}^{+}$line has been detected previously (see Harju et al. 2006, and references therein). The masses, sizes, relatively high degree of deuteration and the line parameters of the condensations indicate that they are low- to intermediatemass dense cores (cf. Fontani et al. 2008). IRAS 05405-0117 and SMM 4 are likely to represent Class 0 protostellar cores (see Sect. 5.1), whereas the subsidiary cores, e.g., Ori B9 N, are in an earlier, prestellar phase.

\section{Summary and conclusions}

We have mapped the Ori B9 cloud in the $870 \mu \mathrm{m}$ dust continuum emission with the APEX telescope. We have also observed $\mathrm{N}_{2} \mathrm{H}^{+}(1-0)$ and $\mathrm{N}_{2} \mathrm{D}^{+}(2-1)$ spectral line emission towards selected positions in Ori B9 with the IRAM $30 \mathrm{~m}$ telecope. These observations have been used together with archival Spitzer/MIPS data to derive the physical characteristics of the cores in Ori B9 and the degree of deuterium fractionation and

12 Harju et al. assumed that $x\left(\mathrm{NH}_{3}\right) \sim 3 \times 10^{-8}$. Using the $\mathrm{H}_{2}$ column densities from the dust continuum, we derived the values of $x\left(\mathrm{NH}_{3}\right) \sim$ $1-2 \times 10^{-7}$ from our line observation positions. ionization degree within the IRAS 05405-0117 clump region. The main results of our work are:

1. The LABOCA field contains 12 compact submm sources. Four of them are previously known IRAS sources, and eight of them are new submm sources. All the IRAS sources and two of the new submm sources are associated with the Spitzer 24 and $70 \mu \mathrm{m}$ sources. The previously unknown sources, SMM 3 and SMM 4, are promising Class 0 candidates based on their SEDs between 24 and $870 \mu \mathrm{m}$. There is an equal number of starless and protostellar cores in the cloud. We suggest that the majority of our starless cores are likely to be prestellar because of their high densities.

2. The total mass of the cloud as estimated from the 2MASS near-infrared extinction map is $1400 M_{\odot}$. The submm cores constitute about $3.6 \%$ of the total cloud mass. This percentage agrees with the observed low values of star-formation efficiency in nearby molecular clouds.

3. The mass distribution of the cores of Ori B9 and Orion B North studied by Nutter \& Ward-Thompson (2007) probably represent those of subsamples of the same parent distribution. The CMF for the Orion B North is well-matched to the stellar IMF (Goodwin et al. 2008). The core separations in these two regions are also similar, indicating that the fragmentation length-scale is similar. Since the fragmentation length-scales are alike and the CMFs resemble the IMF, the origin of cores could be explained in terms of turbulent fragmentation. The clustered mode of star formation in these two different regions suggests that turbulence is driven on large scales.

4. On average, the starless cores are larger and more elongated than the protostellar cores in Ori B9. The observed mean angular sizes and axis ratios suggest average density-powerlaw indices $p \sim 1$ and $\sim 1.5$ for starless and protostellar cores, respectively.

5. The fractional $\mathrm{N}_{2} \mathrm{H}^{+}$and $\mathrm{N}_{2} \mathrm{D}^{+}$abundances within the clump associated with IRAS 05405-0117 are $\sim 4-11 \times 10^{-10}$ and $2-5 \times 10^{-11}$, respectively. The $N\left(\mathrm{~N}_{2} \mathrm{D}^{+}\right) / N\left(\mathrm{~N}_{2} \mathrm{H}^{+}\right)$column density ratio varies between $0.03-0.04$. This is a typical degree of deuteration in low-mass dense cores and conforms with the earlier detection of $\mathrm{H}_{2} \mathrm{D}^{+}$. There is evidence of a $\mathrm{N}_{2} \mathrm{H}^{+}$"hole" in the protostellar Class 0 candidate SMM 4. The envelope of SMM 4 probably represents an extreme case of depletion where $\mathrm{N}_{2} \mathrm{H}^{+}$has also disappeared from the gas phase.

6. The ionization fraction (electron abundance) in the positions studied is estimated to be $x(\mathrm{e}) \sim 10^{-7}$. There is uncertainty about the most abundant ionic species. The most likely candidates are $\mathrm{H}^{+}$and $\mathrm{HCO}^{+}$. The cosmic-ray ionization rate in the observed positions was found to be $\zeta_{\mathrm{H}_{2}} \sim$ $1-2 \times 10^{-16} \mathrm{~s}^{-1}$.

7. There seems to be a discrepancy between the chemical age derived near IRAS 05405-0117 and the statistical age deduced from the numbers of starless and protostellar cores, which suggests that the duration of the prestellar phase of core evolution is comparable to the free-fall time. The statistical age estimate is, however, likely to be biased because the cores detected in this survey are rather dense $\left(n\left(\mathrm{H}_{2}\right) \gtrsim\right.$ $10^{5} \mathrm{~cm}^{-3}$ ) and thus represent the most advanced stages of evolution.

Acknowledgements. We thank the referee, Paola Caselli, for her insightful comments and suggestions that helped to improve the paper. The authors are grateful to the sfaff of the IRAM $30 \mathrm{~m}$ telescope, for their hospitality and help during the 
observations. We also thank the staff at the APEX telescope site. We are very grateful to Edouard Hugo, Oskar Asvany, and Stephan Schlemmer for making available their rate coefficients of the reaction $\mathrm{H}_{3}^{+}+\mathrm{H}_{2}$ with deuterated isotopologues. O.M. acknowledges Martin Hennemann for providing the SED fitting tool originally written by Jürgen Steinacker, and the Research Foundation of the University of Helsinki. The team acknowledges support from the Academy of Finland through grant 117206. This work is based in part on observations made with the Spitzer Space Telescope, which is operated by the Jet Propulsion Laboratory, California Institute of Technology under a contract with NASA.

\section{References}

Aikawa, Y., Herbst, E., Roberts, H., \& Caselli, P. 2005, ApJ, 620, 330

Aikawa, Y., Wakelam, V., Garrod, R. T., \& Herbst, E. 2008a, ApJ, 674, 984

Aikawa, Y., Wakelam, V., Sakai, N., et al. 2008b, in Organic Matter in Space, ed. S. Kwok, \& S. Sandford, Proc. IAU, 251, 129

André, P., Belloche, A., Motte, F., \& Peretto, N. 2007, A\&A, 472, 519

André, P., Basu, S., \& Inutsuka, S. 2008, in Structure Formation in Astrophysics, ed. G. Chapier (Cambridge Univ. Press)

Bacmann, A., Lefloch, B., Ceccarelli, C., et al. 2002, A\&A, 389, L6

Ballesteros-Paredes, J., Klessen, R. S., Mac Low, M.-M., \& Vázquez-Semadeni, E. 2007, in Protostars and Planets V, ed. B. Reipurth, D. Jewitt, \& K. Keil (Tucson: Univ. of Arizona Press), 63

Bally, J., Reipurth, B., \& Aspin, C. 2002, ApJ, 574, L79

Belloche, A., \& André, P. 2004, A\&A, 419, L35

Bergin, E. A., \& Langer, W. D. 1997, ApJ, 486, 316

Bergin, E. A., Plume, R., Williams, J. P., \& Myers, P. C. 1999, ApJ, 512, 724

Bergin, E. A., Alves, J., Huard, T., \& Lada, C. J. 2002, ApJ, 570, L101

Bohlin, R. C., Savage, B. D., \& Drake, J. F. 1978, ApJ, 224, 132

Bontemps, S., André, P., Terebey, S., \& Caprit, S. 1996, A\&A, 311, 858

Burke, J. R., \& Hollenbach, D. J. 1983, ApJ, 265, 223

Cabrit, S., Codella, C., Gueth, F., et al. 2007, A\&A, 468, L29

Caselli, P. 2002, P\&SS, 50, 1133

Caselli, P., \& Myers, P. C. 1994, in Clouds, Cores, and Low Mass Stars, ASP Conf. Ser., 65, 52

Caselli, P., \& Myers, P. C. 1995, ApJ, 446, 665

Caselli, P., Myers, P. C., \& Thaddeus, P. 1995, ApJ, 455, L77

Caselli, P., Walmsley, C. M., Terzieva, R., \& Herbst, E. 1998, ApJ, 499, 234

Caselli, P., Walmsley, C. M., Tafalla, M., et al. 1999, ApJ, 523, L165

Caselli, P., Walmsley, C. M., Zucconi, A., et al. 2002a, ApJ, 565, 344

Caselli, P., Benson, P. J., Myers, P. C., \& Tafalla, M. 2002b, ApJ, 572, 238

Caselli, P., Vastel, C., Ceccarelli, C., et al. 2008, A\&A, 492, 703

Chen, X., Launhardt, R., Bourke, T. L., et al. 2008, ApJ, 683, 862

Ciolek, G. E., \& Basu, S. 2001, ApJ, 547, 272

Codella, C., Cabrit, S., Gueth, F., et al. 2007, A\&A, 462, L53

Crapsi, A., Caselli, P., Walmsley, C. M., et al. 2004, A\&A, 420, 957

Crapsi, A., Caselli, P., Walmsley, C. M., et al. 2005a, ApJ, 619, 379

Crapsi, A., DeVries, C. H., Huard, T. L., et al. 2005b, A\&A, 439, 1023

Crapsi, A., Caselli, P., Walmsley, M. C., \& Tafalla, M. 2007, A\&A, 470, 221

Crutcher, R. M. 1999, ApJ, 520, 706

Crutcher, R. M., \& Troland, T. H. 2000, ApJ, 537, L139

Crutcher, R. M., Nutter, D. J., Ward-Thompson, D., \& Kirk, J. M. 2004, ApJ, 600, 279

Crutcher, R. M., Hakobian, N., \& Troland, T. H. 2009, ApJ, 692, 844

Daniel, F., Cernicharo, J., \& Dubernet, M.-L. 2006, ApJ, 648, 461

Daniel, F., Cernicharo, J., Roueff, E., Gerin, M., \& Dubernet, M.-L. 2007, ApJ, 667,980

Dore, L., Caselli, P., Beninati, S., et al. 2004, A\&A, 413, 1177

Draine, B. T., \& Sutin, B. 1987, ApJ, 320, 803

Emprechtinger, M., Caselli, P., Volgenau, N. H., et al. 2009, A\&A, 493, 89 Engelbracht, C. W., Blaylock, M., Su, K. Y. L., et al. 2007, PASP, 119, 994 Enoch, M. L., Glenn, J., Evans II, N. J., et al. 2007, ApJ, 666, 982 Enoch, M. L., Evans II, N. J., Sargent, A. I., et al. 2008, ApJ, 684, 1240 Flower, D. R. 2000, MNRAS, 313, L19

Flower, D. R., Pineau des Forêts, G., \& Walmsley, C. M. 2005, A\&A, 436, 933 Flower, D. R., Pineau des Forêts, G., \& Walmsley, C. M. 2006a, A\&A, 449, 621 Flower, D. R., Pineau des Forêts, G., \& Walmsley, C. M. 2006b, A\&A, 456, 215 Flower, D. R., Pineau des Forêts, G., \& Walmsley, C. M. 2007, A\&A, 474, 923 Fontani, F., Caselli, P., Crapsi, A., et al. 2006, A\&A, 460, 709

Fontani, F., Caselli, P., Bourke, T. L., Cesaroni, R., \& Brand, J. 2008, A\&A, 477, L45

Frerking, M. A., Langer, W. D., \& Wilson, R. W. 1982, ApJ, 262, 590

Froebrich, D. 2005, ApJS, 156, 169
Galván-Madrid, R., Vázquez-Semadeni, E., Kim, J., \& Ballesteros-Paredes, J. 2007, ApJ, 670, 480

Geppert, W. D., Thomas, R., Semaniak, J., et al. 2004, ApJ, 609, 459

Gerin, M., Pearson, J. C., Roueff, E., et al. 2001, ApJ, 551, L193

Gerlich, D., Herbst, E., \& Roueff, E. 2002, P\&SS, 50, 1275

Goodwin, S. P., Nutter, D., Kroupa, P., et al. 2008, A\&A, 477, 823

Gordon, K. D., Engelbracht, C. W., Fadda, D., et al. 2007, PASP, 119, 1019

Harju, J., Walmsley, C. M., \& Wouterloot, J. G. A. 1993, A\&AS, 98, 51

Harju, J., Haikala, L. K., Lehtinen, K., et al. 2006, A\&A, 454, L55

Hatchell, J., Fuller, G. A., Richer, J. S., et al. 2007, A\&A, 468, 1009

Havenith, M., Zwart, E., Leo Meerts, W., \& Ter Meulen, J. J. 1990, J. Chem. Phys., 93, 8446

Hotzel, S., Harju, J., \& Walmsley, C. M. 2004, A\&A, 415, 1065

Hugo, E., Asvany, E., \& Schlemmer, S. 2009, J. Chem. Phys., in press

Kirk, H., Johnstone, D., \& Tafalla, M. 2007, ApJ, 668, 1042 Klessen, R. S. 2001, ApJ, 556, 837

Lacy, J. H., Knacke, R., Geballe, T. R., \& Tokunaga, A. T. 1994, ApJ, 428, L69 Lada, E. A. 1992, ApJ, 393, L25

Lada, E. A., Bally, J., \& Stark, A. A. 1991, ApJ, 368, 432

Launhardt, R., Mezger, P. G., Haslam, C. G. T., et al. 1996, A\&A, 312, 569

Lee, J.-E., Evans, N. J., II, Shirley, Y. L., \& Tatematsu, K. 2003, ApJ, 583, 789

Lee, C.-F., Ho, P. T. P., Beuther, H., et al. 2006, ApJ, 639, 292

Lee, C.-F., Ho, P. T. P., Hirano, N., et al. 2007, ApJ, 659, 499

Linsky, J. L., Diplas, A., Wood, B. E., et al. 1995, ApJ, 451, 335

Linsky, J. L., Draine, B. T., Moos, H. W., et al. 2006, ApJ, 647, 1106

Lombardi, M., \& Alves, J. 2001, A\&A, 337, 1023

Mac Low, M.-M., \& Klessen, R. S. 2004, Rev. Mod. Phys., 76, 125

Mathis, J. S., Rumpl, W., \& Nordsieck, K. H. 1977, ApJ, 217, 425

McKee, C. F. 1989, ApJ, 345, 782

McKee, C. F., Zweibel, E. G., Goodman, A. A., \& Heiles, C. 1993, in Protostars and Planets III, ed. E. Levy, \& J. Lunine (Tucson: Univ. of Arizona Press), 327

Megeath, S. T., Li, Z.-Y., \& Nordlund, Å. 2008, in Structure formation in the Universe [arXiv: 0801.0492]

Mouschovias, T. Ch., \& Spitzer, L. Jr. 1976, ApJ, 210, 326

Myers, P. C., Adams, F. C., Chen, H., \& Schaff, E. 1998, ApJ, 492, 703

Nutter, D., \& Ward-Thompson, D. 2007, MNRAS, 374, 1413

Offner, S. S. R., \& Krumholz, M. R. 2009, ApJ, 693, 914

Oliveira, C. M., Hébrard, G., Howk, J. C., et al. 2003, ApJ, 587, 235

Ossenkopf, V., \& Henning, Th. 1994, A\&A, 291, 943

Padoan, P., Willacy, K., Langer, W., \& Juvela, M. 2004, ApJ, 614, 203

Pagani, L., Salex, M., \& Wannier, P. G. 1992, A\&A, 258, 479

Pagani, L., Pardo, J.-R., Apponi, A. J., et al. 2005, A\&A, 429, 181

Pagani, L., Bacmann, A., Cabrit, S., \& Vastel, C. 2007, A\&A, 467, 179

Pagani, L., Vastel, C., Hugo, E., et al. 2009a, A\&A, 494, 623

Pagani, L., Daniel, F., \& Dubernet, M.-L. 2009b, A\&A, 494, 719

Palau, A., Estatella, R., Girart, J. M., et al. 2007, A\&A, 465, 219

Rathborne, J. M., Lada, C. J., Muench, A. A., et al. 2009, ApJ, in press

Roberts, H., \& Millar, T. J. 2007, A\&A, 471, 849

Roberts, H., Herbst, E., \& Millar, T. J. 2003, ApJ, 591, L41

Roberts, H., Herbst, E., \& Millar, T. J. 2004, A\&A, 424, 905

Sault, R. J., Teuben, P. J., \& Wright, M. C. H. 1995, in Astronomical Data Analysis Software and Systems IV, ed. R. Shaw, H. E. Payne, \& J. J. E. Hayes, ASP Conf. Ser., 77, 433

Schnee, S., Caselli, P., Goodman, A., et al. 2007, ApJ, 671, 1839

Shirley, Y. L., Nordhaus, M. K., Grcevich, J. M., et al. 2005, ApJ, 632, 982

Shu, F. H., Adams, F. C., \& Lizano, S. 1987, ARA\&A, 25, 23

Simpson, R. J., Nutter, D., \& Ward-Thompson, D. 2008, MNRAS, 391, 205

Smith, M. D., O'Connell, B., \& Davis, C. J. 2007, A\&A, 466, 565

Swift, J. J., \& Williams, J. P. 2008, ApJ, 679, 552

Tafalla, M., Myers, P. C., Caselli, P., et al. 2002, ApJ, 569, 815

Tafalla, M., Myers, P. C., Caselli, P., \& Walmsley, C. M. 2004, A\&A, 416, 191

Tafalla, M., Santiago-García, J., Myers, P. C., et al. 2006, A\&A, 455, 577

Tassis, K., \& Mouschovias, T. Ch. 2004, ApJ, 616, 283

Troland, T. H., \& Crutcher, R. M. 2008, ApJ, 680, 457

Troland, T. H., Crutcher, R. M., Goodman, A. A., et al. 1996, ApJ, 471, 302

Turner, B. E., \& Heiles, C. 2006, ApJS, 162, 388

Walmsley, C. M., Flower, D. R., \& Pineau des Forêts, G. 2004, A\&A, 418, 1035 Ward-Thompson, D., Motte, F., \& André, P. 1999, MNRAS, 305, 143

Ward-Thompson, D., André, P., Crutcher, R., et al. 2007, in Protostars and Planets V, ed. B. Reipurth, D. Jewitt, \& K. Keil (Tucson: Univ. of Arizona Press), 33

Williams, J. P., Bergin, E. A., Caselli, P., et al. 1998, ApJ, 503, 689

Young, C. H., Shirley, Y. L., Evans II, N. J., \& Rawlings, J. M. C. 2003, ApJS, 145,111 